\title{
Interaction of gelatin with polyenes modulates antifungal activity and biocompatibility of electrospun fiber mats
}

This article was published in the following Dove Press journal:

International Journal of Nanomedicine

23 May 2014

Number of times this article has been viewed

\section{Rajamani \\ Lakshminarayanan ${ }^{1,2}$ \\ Radhakrishnan Sridhar ${ }^{3,4}$ \\ Xian Jun Loh ${ }^{5}$ \\ Muruganantham \\ Nandhakumar' \\ Veluchamy Amutha \\ Barathi ${ }^{1,6}$ \\ Madhaiyan Kalaipriya ${ }^{3,4}$ \\ Jia Lin Kwan' \\ Shou Ping Liu',2 \\ Roger Wilmer Beuerman 1,2 \\ Seeram Ramakrishna ${ }^{3,4,7}$ \\ 'Singapore Eye Research Institute, \\ ${ }^{2}$ Signature Research Program in \\ Neuroscience and Behavioral \\ Disorders, Duke-NUS Graduate \\ Medical School, ${ }^{3}$ Department of \\ Mechanical Engineering, National \\ University of Singapore, ${ }^{4}$ Center for \\ Nanofibers and Nanotechnology, \\ National University of Singapore, \\ ${ }^{5}$ Institute of Materials Research and \\ Engineering, A*STAR (Agency for \\ Science, Technology and Research), \\ 3 Research Link, Singapore, \\ ${ }^{6}$ Department of Ophthalmology, Yong \\ Loo Lin School of Medicine, National \\ University of Singapore, ${ }^{7} \mathrm{NUS}$ \\ Nanoscience and Nanotechnology \\ Initiative, Singapore}

Correspondence: Rajamani

Lakshminarayanan

Singapore Eye Research Institute,

II Third Hospital Avenue,

Singapore I6875 I

Email lakshminarayanan.rajamani@seri. com.sg
Abstract: Topical application of antifungals does not have predictable or well-controlled release characteristics and requires reapplication to achieve therapeutic local concentration in a reasonable time period. In this article, the efficacy of five different US Food and Drug Administration-approved antifungal-loaded (amphotericin B, natamycin, terbinafine, fluconazole, and itraconazole) electrospun gelatin fiber mats were compared. Morphological studies show that incorporation of polyenes resulted in a two-fold increase in fiber diameter and the mats inhibit the growth of yeasts and filamentous fungal pathogens. Terbinafine-loaded mats were effective against three filamentous fungal species. Among the two azole antifungals compared, the itraconazole-loaded mat was potent against Aspergillus strains. However, activity loss was observed for fluconazole-loaded mats against all of the test organisms. The polyene-loaded mats displayed rapid candidacidal activities as well. Biophysical and rheological measurements indicate strong interactions between polyene antifungals and gelatin matrix. As a result, the polyenes stabilized the triple helical conformation of gelatin and the presence of gelatin decreased the hemolytic activity of polyenes. The polyene-loaded fiber mats were noncytotoxic to primary human corneal and sclera fibroblasts. The reduction of toxicity with complete retention of activity of the polyene antifungal-loaded gelatin fiber mats can provide new opportunities in the management of superficial skin infections.

Keywords: fungal infections, electrospinning, antifungals, controlled release, drug-matrix interactions

\section{Introduction}

Pathogens from a large group of fungi and yeasts are increasingly impacting the global biota and constitute one of the planet's biggest killers threatening food security and ecosystems. ${ }^{1-3}$ While superficial fungal infections are the most common form of infections, affecting $20 \%-25 \%$ of the world's population, ${ }^{4}$ the invasive fungal and yeast infections associated with excessive use of medical devices and implants and expanding numbers of immunocompromised patients are responsible for increased health care costs, morbidity, and mortality. ${ }^{5-7}$ In addition, new fungal and yeast pathogens have emerged that have reduced susceptibility or enhanced resistance to the available antifungals. ${ }^{8-10}$ Compared to antibiotics for treating bacterial infections, only limited categories of antifungal drugs are available for fungal infections: the polyene macrolides, azoles, allylamines, fluoropyrimidines, and echinocandins. ${ }^{11,12}$

Due to warm and humid conditions in tropical countries, superficial and subcutaneous fungal infections are relatively common. ${ }^{13}$ The architecture of the skin presents a formidable barrier for drug penetration, thus impeding the drug efficacy and 
local bioavailability. ${ }^{14}$ Therefore, a high concentration of the drug at the site of infection is required to achieve clinical efficacy. A number of strategies such as polymeric and peptide hydrogels, nanoparticles, microemulsion, liposomes, niosomes, or ethosomes that can function as drug depots for delivering the antifungals at the site of infections have been reported. ${ }^{15-20}$ However, these formulations need to be applied frequently to maintain sustained release of the drugs. ${ }^{21}$ Nonwoven fiber mats produced by electrospinning have the potential for a wide range of applications such as filters, composites, drug carriers, wound dressing, and tissue regeneration..$^{22-25}$ Wound dressings prepared by electrospinning of biopolymers provide several key attributes, such as high encapsulation efficiency and sustained delivery of drugs for a longer time, that are required for transdermal/topical delivery of drugs. ${ }^{26}$ Electrospun fiber mats carrying various antimicrobial agents, carbon nanotubes, N-halamines, vitamin $\mathrm{A}$, and vitamin $\mathrm{E}$ have been reported. ${ }^{27-32}$ However, the in vitro efficacy of antifungal drugs incorporated into electrospun fibers against yeasts/filamentous fungi has not yet been demonstrated. ${ }^{33}$

In terms of mechanism of action, the available antifungals can be grouped into three categories: 1) polyene, azole, and allylamine antifungals that target ergosterol or ergosterol biosynthetic pathways; 2 ) echinocandins, which are $\beta$-glucan synthase inhibitors; and 3) fluoropyrimidines, which target RNA synthesis. Due to its inherent biodegradability under physiological environments and nonimmunogenic properties, gelatin is used in numerous food and cosmetic applications as well as in cell-compatible biomaterials. ${ }^{34,35}$ Using appropriate solvent composition, electrospinning has been used to generate gelatin fibers with a wide range of diameters. ${ }^{36}$ In this study, the efficacy of polyene, azole, and allylamine antifungal drugs (Figure 1) incorporated into gelatin fiber mats against various yeasts/fungal pathogens were evaluated, their fungicidal kinetics were tested, and their toxicity on primary fibroblast cells was assessed. The interaction between drugs and gelatin was investigated by circular dichroism (CD) and rheological studies.

\section{Materials and methods Chemicals and reagents}

Gelatin (from porcine skin, high bloom), amphotericin B, natamycin, terbinafine hydrochloride, itraconazole, fluconazole, and trifluoroethanol were purchased from SigmaAldrich (St Louis, MO, USA). All chemicals were of analytical grade and used without further purification.

\section{Electrospinning of antifungal-loaded gelatin fiber mats}

Antifungal drug-loaded gelatin fiber mats were fabricated by the electrospinning method. Gelatin ( $1 \mathrm{~g}$ ) was dissolved in $10 \mathrm{~mL}$ of trifluoroethanol at a concentration of $10 \%$ weight/ volume $(w / v)$. The drugs were added to the gelatin solution so that the final antifungal:gelatin ratio was maintained at $0.25 \mathrm{wt} \%$. The mixture was stirred overnight at room
A

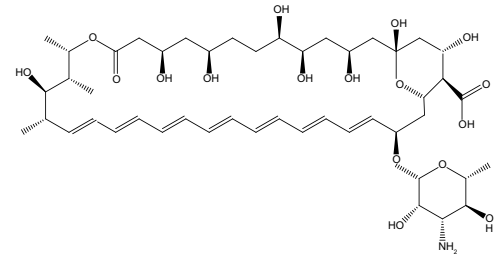

B

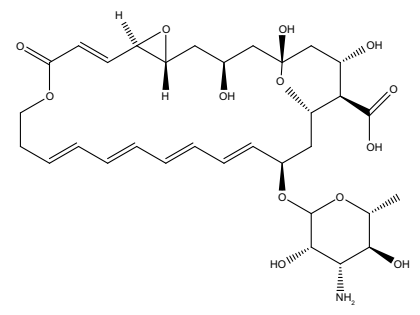

C

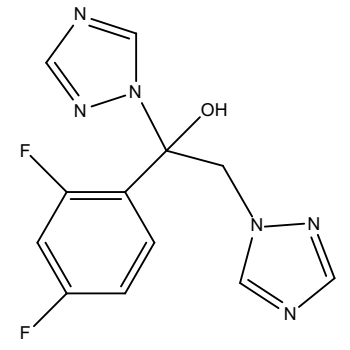

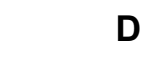

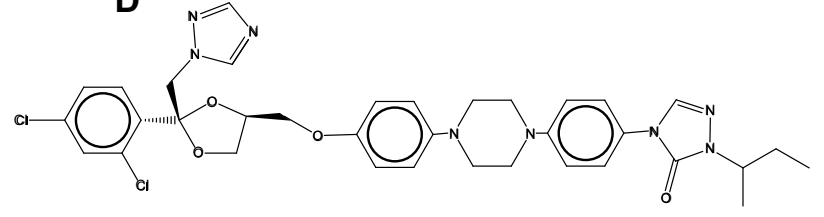

$\mathrm{E}$

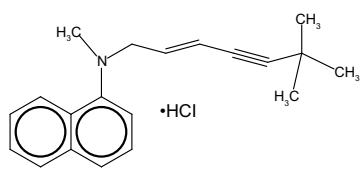

Figure I Chemical structures of antifungals used in this study. (A) Amphotericin B; (B) natamycin; (C) fluconazole; (D) itraconazole; (E) terbinafine chloride. 
temperature to achieve a homogenous solution. The overnight stirred solution was poured into a standard $10 \mathrm{~mL}$ syringe that was attached to a steel needle. Electrospinning was carried out by introducing a high voltage (direct current high voltage power supply; Gamma High Voltage Research, Inc., Ormond Beach, FL, USA) to the needle as a positive electrode and a fixed sheet of grounded aluminum foil. The distance between the needle tip and collector was set at 12 $\mathrm{cm}$. When the positive electrode reached a critical voltage of $12 \mathrm{kV}$, the spinning head extruded polymer solution and the electrospun fibers were deposited on to the aluminum foil kept at the collector. The emitting rate of the polymer solution was controlled at $0.6 \mathrm{~mL} /$ hour by means of a syringe pump (KD Scientific Inc., Holliston, MA, USA). The as-spun fiber mats were exposed for vapor phase crosslinking of glutaraldehyde by placing an aqueous solution of glutaraldehyde $(50 \%)$ in a Petri dish kept beneath the fiber mats in a closed desiccator. After glutaraldehyde vapor treatment, the sample was vacuum dried for 2 days to remove residual glutaraldehyde and solvent. The crosslinking time was 3 hours and 24 hours for the antifungal-loaded fiber mats and control gelatin mats, respectively.

\section{Scanning electron microscopy (SEM)}

The morphology and diameter of the gelatin crosslinked electrospun fibers were observed and determined with the use of an optical microscope (BX51M; Olympus Corporation, Tokyo, Japan) and an SEM (Quanta 200F; FEI, Hillsboro, Oregon, USA). Prior to imaging with the use of SEM, a small section of the fibers on the sample holder was sputter coated with gold (JFC-1200 fine coater; JEOL, Tokyo, Japan). SEM was then used to observe the samples at an accelerating voltage of $10-15 \mathrm{kV}$. The average diameter of the fibers $(n=50)$ was estimated from four SEM images, each from two independent experiments.

\section{Radial diffusion assay}

Fungus and yeast cell cultures (at a concentration of 0.5 McFarland standards) were spread onto the surface of sterile Sabouraud dextrose agar plates using a cotton swab in $9 \mathrm{~cm}$ diameter Petri dishes. The fiber mats $(0.5 \mathrm{~cm} \times 0.5 \mathrm{~cm})$ and antifungal-loaded gelatin fiber mats were placed on top of the swabbed cultures and incubated at $37^{\circ} \mathrm{C}$. Antifungal activity of fiber mats was visualized as the diameter of the zone of inhibition after incubating plates in the dark for 48 hours for Candida strains and 72 hours for Fusarium and Aspergillus strains. The assay was performed in two independent duplicates and the average value was reported.

\section{Kinetics of candidacidal action}

To assess the candidacidal properties of the polyene-loaded fiber mats, time-kill kinetics against a Candida albicans DF 2672R strain was carried out. Cultures were grown overnight in Sabouraud dextrose broth and the yeast cell concentration was adjusted to $1.5 \times 10^{5}$ Colony Forming Units $(\mathrm{CFU}) / \mathrm{mL}$. Fiber mats and antifungal-loaded fiber mats $(0.5 \times 0.5 \mathrm{~cm}$ in triplicates $)$ were incubated with $1 \mathrm{~mL}$ of the cell suspension in a 24-well Nunc ${ }^{\circledR}$ dish (Thermo Fisher Scientific, Waltham, MA, USA) and incubated at $37^{\circ} \mathrm{C}$ with constant shaking. At predetermined time points, $100 \mu \mathrm{L}$ of the suspension was withdrawn, serially diluted $\left(10^{2}\right.$ or $10^{3}$ fold), and poured into a Sabouraud dextrose agar plate. The plate was incubated for 48 hours at $37^{\circ} \mathrm{C}$ for colony counting. The data were expressed in terms of percentage of fungal survival with respect to the positive control (ie, fiber mats without antifungals).

\section{Antifungals release properties of electrospun fiber mats}

The antifungal-loaded fiber mats $(n=4)$ were weighed and immersed in $10 \mathrm{~mL}$ of phosphate buffered saline (PBS) buffer ( $\mathrm{pH} 7.0$ ) with constant shaking at $37^{\circ} \mathrm{C}$. A $1 \mathrm{~mL}$ aliquot of the above solution was withdrawn at various time intervals, centrifuged, and the amount of drugs in the supernatant was determined by spectrophotometry with the calibration method. Each time the mother liquor was reloaded with a fresh $1 \mathrm{~mL}$ of buffer to maintain the total volume of the release solution at $10 \mathrm{~mL}$. The characteristic absorption bands of each antifungal were used to estimate the amounts of antifungals released. The values were converted into $\mu \mathrm{g}$ of antifungals released/10 $\mathrm{mg}$ of the fiber mat.

\section{Minimum inhibitory concentration (MIC) of antifungals}

MIC values for amphotericin B, natamycin, fluconazole, and terbinafine chloride were determined in two independent duplicates by the microdilution method. Overnight cultivated yeast strains were suspended in Sabouraud dextrose broth at a starting optical density at $600 \mathrm{~nm}$ of $\sim 0.08$ in a flat-bottomed microtiter plate. A serial dilution of the antifungals in the same broth was mixed with the inoculum to give a final peptide concentration of $0.1-128 \mu \mathrm{g} / \mathrm{mL}$. The antifungal activity was assessed by monitoring the optical density at $600 \mathrm{~nm}$ in cycles of 30 minutes and an orbital shaking at $100 \mathrm{rpm}$ using an Infinite ${ }^{\circledR}$ M200 microplate reader (Tecan Group Ltd, Männedorf, Switzerland) for 48 hours at $37^{\circ} \mathrm{C}$. Cultures without antifungals were used as positive controls 
and broth alone or with $256 \mu \mathrm{g} / \mathrm{mL}$ of the antifungals served as negative controls. The minimum concentration required for complete inhibition was assessed by both visible observations as well as by measuring the optical density at $600 \mathrm{~nm}$ and taken as the MIC.

\section{CD spectropolarimetry}

Far ultraviolet (UV) CD spectra of gelatin $(0.1 \mathrm{mg} / \mathrm{mL})$ was recorded on a spectropolarimeter (J810; JASCO International Co, Ltd, Tokyo, Japan) in $10 \mathrm{mM}$ PBS (pH 7.0) using a $0.1 \mathrm{~cm}$ path length quartz cuvette at $30^{\circ} \mathrm{C}$. Spectra were recorded from $260 \mathrm{~nm}$ to $190 \mathrm{~nm}$ (for gelatin) and $450 \mathrm{~nm}$ to $300 \mathrm{~nm}$ (for amphotericin B) in $0.1 \mathrm{~nm}$ steps at a scan rate of $50 \mathrm{~nm} /$ minute. The final spectrum is the average of four scans. For the variable temperature $\mathrm{CD}$ experiments, ellipticity at $224 \mathrm{~nm}$ was monitored over a temperature of $10^{\circ} \mathrm{C}-60^{\circ} \mathrm{C}$ at a heating rate of $5^{\circ} \mathrm{C} /$ minute. To study the influence of antifungals on the secondary structure, gelatin was incubated with $0.25 \mathrm{wt} \%$ antifungals and the $\mathrm{CD}$ measurements were recorded as before.

\section{Rheological characterization of antifungal-gelatin interactions}

Rheological experiments were performed at room temperature using a controlled strain rheometer (ARES-G2; TA Instruments, New Castle, DE, USA). The rheometer is equipped with two sensitive force transducers for torque ranging from $0.05 \mathrm{mN} / \mathrm{m}$ to $200 \mathrm{mN} / \mathrm{m}$. The gel was placed onto parallel-plate geometry (25 $\mathrm{mm}$ in diameter). The dynamic storage modulus $\left(\mathrm{G}^{\prime}\right)$ and loss modulus $\left(\mathrm{G}^{\prime \prime}\right)$ of the gel were examined as a function of frequency from $0.1-100 \mathrm{rad} / \mathrm{second}$. The measurements were carried out at strain amplitude $(\gamma 0)$ of $5 \%$ to ensure the linearity of viscoelasticity.

\section{UV spectroscopy of amphotericin B}

UV spectra of amphotericin B were recorded on a UV1800 double beam spectrophotometer (Shimadzu, Kyoto, KYT, Japan) at room temperature. To determine aggregation status of amphotericin B, the spectra were recorded in dimethyl sulfoxide (DMSO), $10 \mathrm{mM}$ PBS buffer ( $\mathrm{pH} 7.0$ ), and in the presence of gelatin.

\section{Hemolytic activity of polyenes in the presence of gelatin}

Hemolytic activity of polyenes in the presence of gelatin was determined against rabbit red blood cells. ${ }^{37}$ Briefly, two independent triplicates of $32 \mu \mathrm{g} / \mathrm{mL}$ and $64 \mu \mathrm{g} / \mathrm{mL}$ of amphotericin B or natamycin in PBS was mixed with rabbit red blood cells to a final concentration of $4 \%$ volume/volume $(\mathrm{v} / \mathrm{v})$, incubated at $37^{\circ} \mathrm{C}$ for 1 hour and centrifuged at 3,000 rpm for 10 minutes. To study the effect of gelatin-antifungal interactions, the drugs were incubated overnight with an appropriate amount of gelatin so that the antifungal:gelatin ratio was $0.25 \mathrm{wt} \%$ and added to the rabbit red blood cells. The release of hemoglobin in the supernatant was monitored by measuring the hemoglobin absorbance at $576 \mathrm{~nm}$. The readings from cell suspension in PBS (without any additives) or $1 \%$ Triton-X100 were used as $0 \%$ or $100 \%$ hemolysis, respectively.

\section{Cytotoxicity of antifungal- loaded fiber mats}

Both the antifungal-loaded and bare fiber mats were adhered to microscope cover slips ( $\mathrm{n}=4$ ) and plated onto 24-well Nunc dishes. Human corneal and sclera fibroblasts were grown in HyClone $^{\circledR}$ Dulbecco's Modified Eagle's Medium (Thermo Fisher) with $10 \%$ fetal bovine serum antimycotic solution containing 500 units $/ \mathrm{mL}$ penicillin $\mathrm{G}, 0.1 \mathrm{mg} / \mathrm{mL}$ streptomycin sulfate, and $2.25 \mu \mathrm{g} / \mathrm{mL}$ amphotericin B (Sigma-Aldrich). The cells were seeded at a concentration of $\sim 4 \times 10^{4}$ cells $/$ well and incubated with the fiber mats at $37^{\circ} \mathrm{C}$ for 24 hours. After equilibrating the plates to room temperature, an equal volume of CellTiter-Glo ${ }^{\circledR}$ reagent was added (Promega Corporation, Madison, WI, USA). The adenosine triphosphate content was determined by recording the luminescence on a Tecan Infinity M200 plate reader as per the manufacturer's instruction.

\section{Uniaxial tensile tests}

Mechanical properties of electrospun fibrous membranes were determined with a tabletop uniaxial testing machine (Model 3345; Instron, Norwood, MA, USA) with the use of a $10 \mathrm{~N}$ load cell under a crosshead speed of $5 \mathrm{~mm} /$ minute at ambient conditions $\left(25^{\circ} \mathrm{C}\right.$ and $70 \%$ relative humidity). All samples were prepared in the form of rectangular shape with dimensions of $20 \times 10 \mathrm{~mm}$ from the electrospun fibrous membranes. The thicknesses of samples were measured with a digital micrometer. At least five samples were tested for each type of electrospun fiber mats and the average was reported.

\section{Statistical analysis}

For statistical analysis, the data were analyzed by using Prism $^{\circledR} 6.0$ software (GraphPad Software, Inc., La Jolla, CA, USA). All the experimental data were expressed as mean \pm standard deviation. Two-tailed Student's $t$-test and one-way analysis of variance with Newman-Keuls post hoc analysis 
were used for determining the significance of differences. $P<0.05$ was considered significant.

\section{Results and discussion \\ Morphology of antifungal- loaded fiber mats}

Gelatin and antifungal-loaded gelatin fiber mats were electrospun from trifluoroethanol solution and their antifungal activities were evaluated. After an initial optimization, $10 \% \mathrm{w} / \mathrm{v}$ of gelatin was used and the final antifungal concentration was maintained at $0.25 \%$ weight $/$ weight $(w / w)$ in order to minimize bead formation. Figure $2 \mathrm{~A}-\mathrm{F}$ show the morphology of gelatin and various antifungal-loaded gelatin fibers imaged after glutaraldehyde crosslinking. The images show smooth surface and absence of any beading, pores, or physical defects in various antifungal loaded fiber mats. The average diameters of individual fibers in various antifungal-loaded mats are shown in Figure 2G. Fiber mats without antifungals had a mean diameter of $0.8 \pm 0.4 \mu \mathrm{m}$. The average diameters of the individual fibers in both the polyene antifungal-loaded mats had a larger and broader diameter distribution compared to the other antifungal-loaded mats $(P<0.001)$. For the two polyene-loaded mats, the average diameters of the fibers remained higher for natamycin $(1.8 \pm 1.0 \mu \mathrm{m})$ compared to amphotericin B $(1.4 \pm 0.9 \mu \mathrm{m})$. For electrospun gelatin, an increase in diameter was observed with an increasing concentration of gelatin or solvent composition. ${ }^{36}$ In the absence of these parameters, the above results indicate significant interactions between the polyenes and gelatin matrix. Among the two azoles, addition of fluconazole did not affect the diameter distribution of the fibers $(0.8 \pm 0.4 \mu \mathrm{m})$, whereas incorporation of itraconazole increased the diameter moderately $(1.2 \pm 0.6 \mu \mathrm{m})$. The average diameter of terbinafine-loaded fibers $(1.4 \pm 0.6 \mu \mathrm{m})$ was larger compared to the control fiber mats.

\section{Antifungal properties of electrospun fiber mats}

The antifungal properties of drug-loaded fiber mats were evaluated by radial disc diffusion assay against a panel of $C$. albicans, and the zone of inhibition was measured (Figures S1-S7). Figure 3 shows the activity of antifungalloaded fiber mats (expressed as the zone of inhibition) against various $C$. albicans strains. The results suggest that the antifungal activity depends on the type of antifungals loaded. Polyene-loaded fiber mats displayed excellent antifungal properties, suppressing the growth of both the American
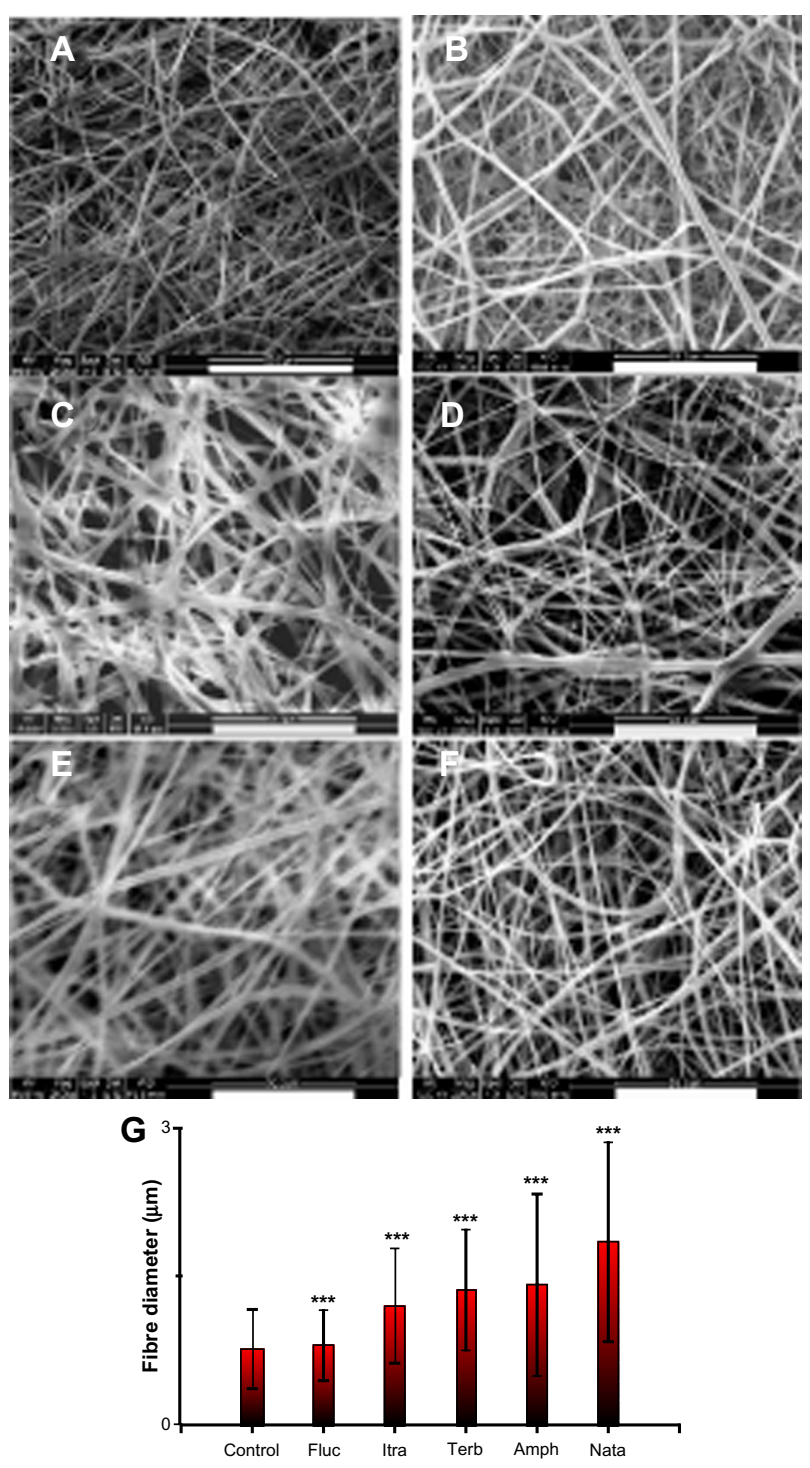

Figure 2 Scanning electron micrographs showing the electrospun gelatin fibers loaded with various antifungals. (A) No antifungals; (B) amphotericin B; (C) natamycin; (D) fluconazole; (E) itraconazole; (F) terbinafine. (G) Histogram showing the effects of various antifungals on the average fiber diameter. Notes: $* * * P<0.00$ I; scale bar $=50 \mu \mathrm{m}$.

Type Culture Collection (Manassas, VA, USA) and the clinical isolates of C. albicans (Figure 3A and B). The other antifungal-loaded fiber mats displayed significant variability in anti-Candida activity. Fluconazole- and terbinafine-loaded fiber mats were the least effective as they showed clear zone of inhibition against only one strain of C. albicans (Figure 3C and D). Itraconazole-loaded fiber mats, on the other hand, inhibited the growth of two American Type Culture Collection strains of C. albicans and a weaker inhibitory activity against the other three strains (Figure 3E).

The in vitro antifungal activities of the fiber mats were determined against four filamentous pathogens as well. As was observed before, both the polyene antifungals inhibited 
A
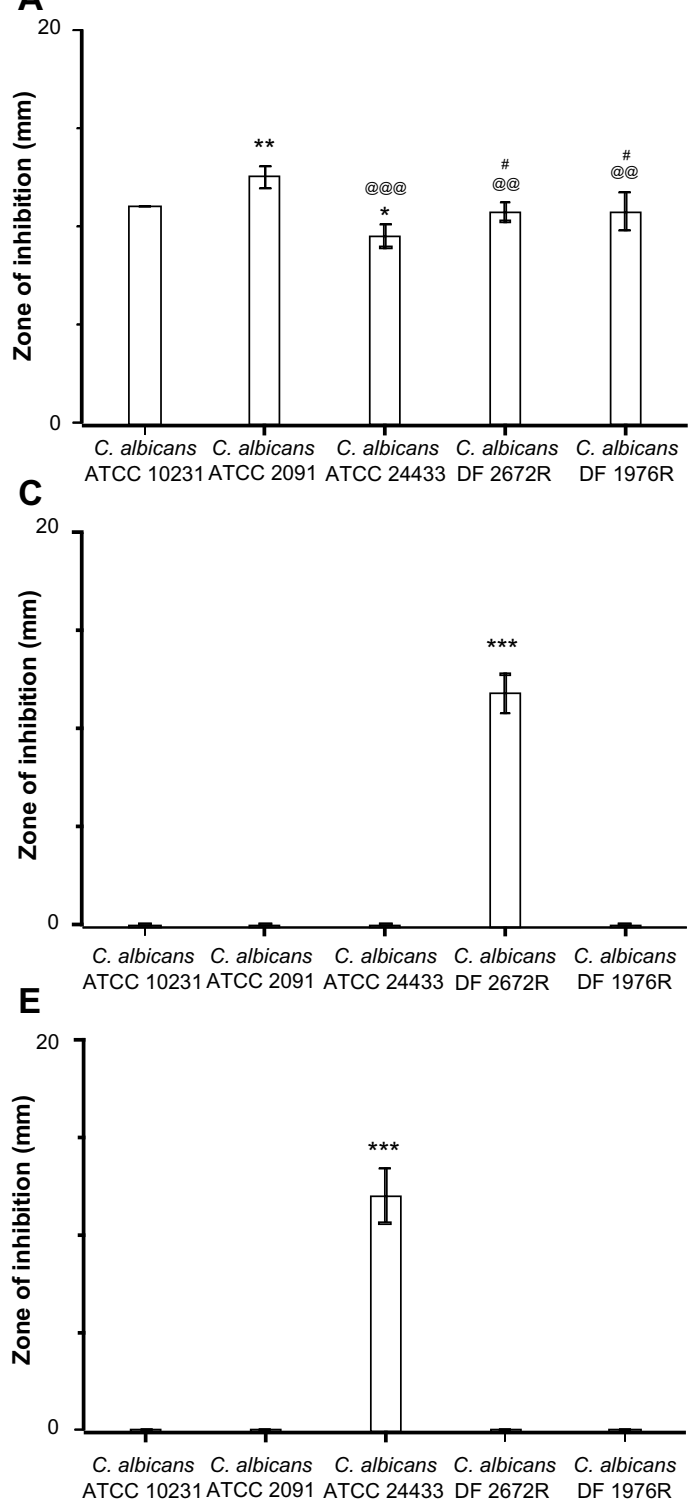

B
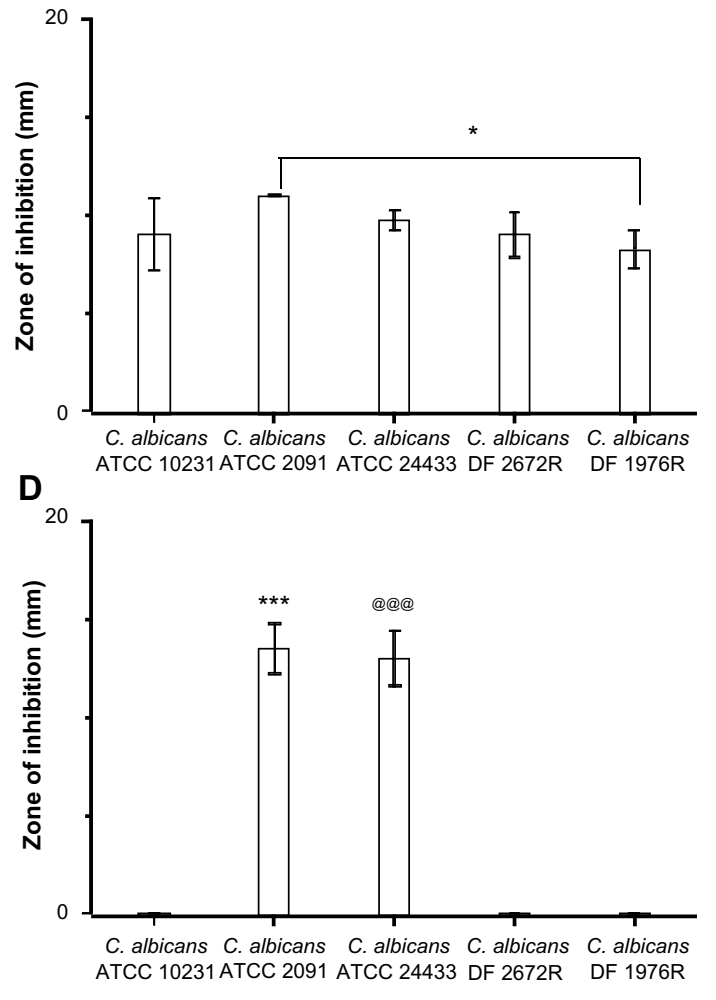

$\mathbf{F}$

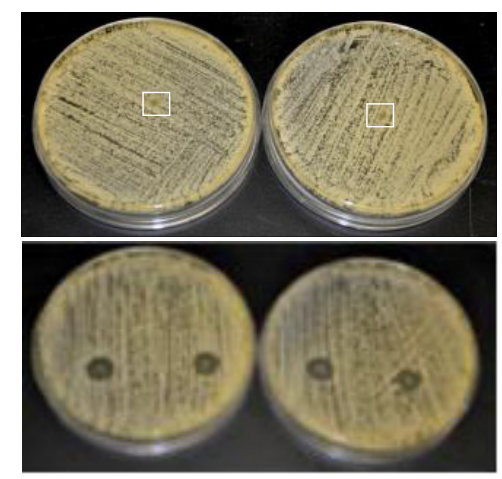

Figure 3 Efficacy of drug-loaded gelatin fiber mats against yeast strains. The antifungal activity against Candida albicans strains is expressed as the zone of inhibition measured by radial diffusion assay. Fiber mats loaded with $(\mathbf{A})$ amphotericin $B(* P<0.05$ compared to $C$. albicans ATCC I023I strains; **P $<0.01$ compared to $C$. albicans ATCC I023I strains; @@P $<0.01$ compared to C. albicans ATCC 209I strains; @@@P $<0.001$ compared to C. albicans ATCC 209I strains; ${ }^{*} P<0.05$ compared to C. albicans ATCC 24433); (B) natamycin ( $* P<0.05$ compared to $C$. albicans ATCC I023I strains); (C) terbinafine ( $* * * P<0.00$ I compared to all the groups); (D) itraconazole ( $* * * P<0.0$ I compared to all the groups except C. albicans ATCC 24433 strains; @@@P $<0.00$ I compared to all the groups except $C$. albicans ATCC 209I strains); and (E) fluconazole. *** $<0.00$ I compared to all the groups. (F) Representative photographs showing the zone of inhibition of gelatin (upper panel) and amphotericin B-loaded gelatin (lower panels) fiber mats.

Note: The absence of bars in the graph indicates no inhibition against the particular strain.

Abbreviation: ATCC, American Tissue Culture Collection.

the growth of all four filamentous fungi (Figure 4A and B). Itraconazole-loaded fiber mats showed strong inhibition for two Aspergillus strains, whereas fluconazole-loaded fiber mats had no activity against all the filamentous pathogens tested (Figure 4D and E). Of all the antifungals, terbinafine chloride-loaded fiber mats displayed the strongest inhibitory activity against filamentous fungal species (Figure 4C). These results suggest that polyene-loaded fiber mats retained the broad-spectrum antifungal properties, whereas terbinafine chloride-loaded fiber mats were effective against filamentous fungi. Among the two azoles, itraconazole-loaded fiber mats were superior compared to fluconazole-loaded fiber mats.

\section{In vitro release of antifungals from gelatin fiber mats and MIC of antifungals}

The cumulative release profile for various antifungals was determined by UV spectrophotometry (Figure 5). The amount of antifungals released is expressed as $\mu \mathrm{g} / \mathrm{mg}$ of fiber mats. 

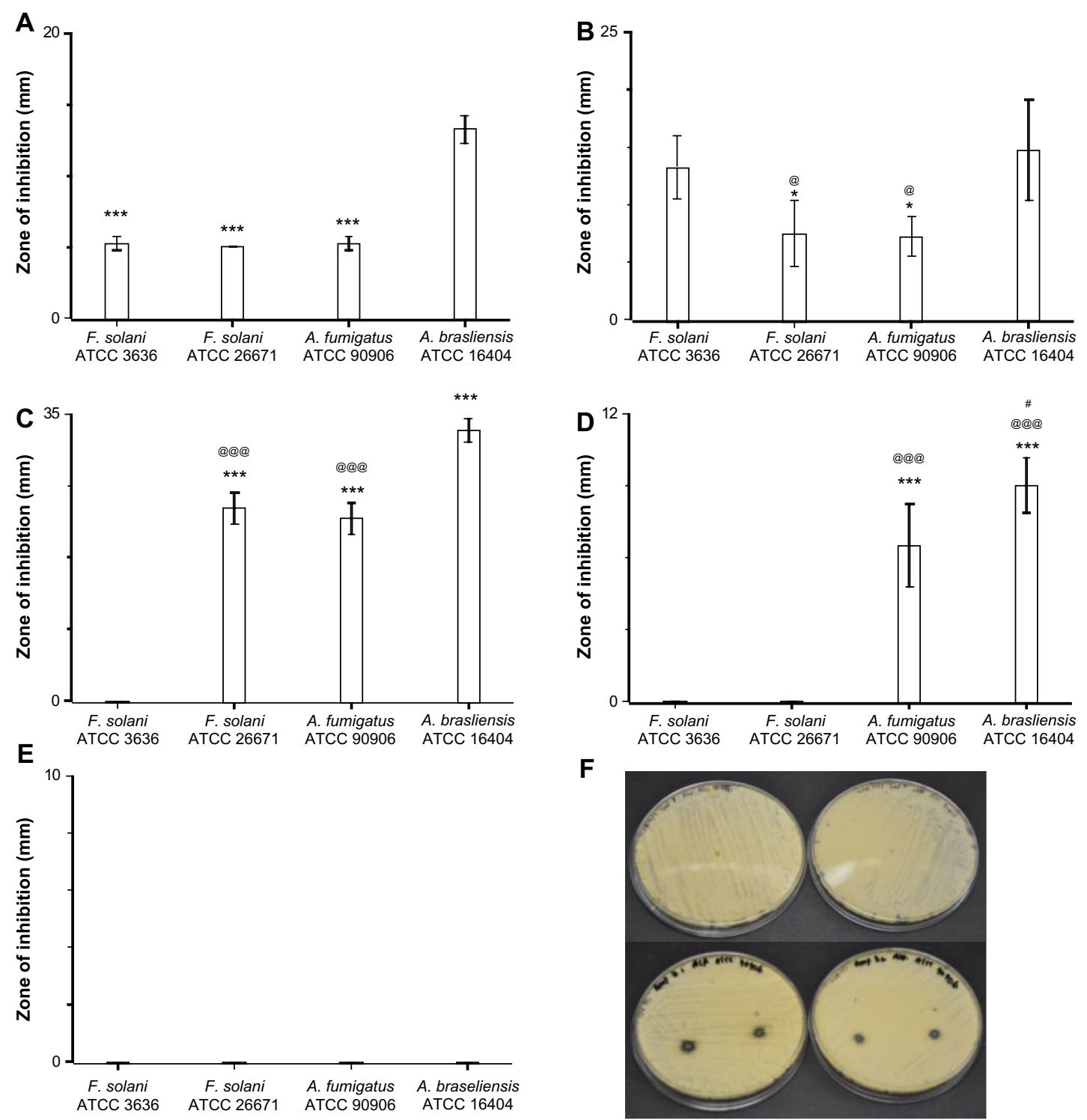

Figure 4 Efficacy of drug-loaded gelatin fiber mats against filamentous fungi. The antifungal activity against Aspergillus and Fusarium strains is expressed as the zone of inhibition measured by radial diffusion assay. Fiber mats loaded with $(\mathbf{A})$ amphotericin $B$ ( $* * * P<0.00$ I compared to Aspergillus brasiliensis ATCC 16404 ); (B) natamycin ( $* P<0.05$ compared to Fusarium solani ATCC 3636 strains; @P $<0.05$ compared to A. brasiliensis ATCC I6404 strains); (C) terbinafine (***P $<0.00$ I compared to F. solani ATCC 3636 strains; @@@ $<0.00$ I compared to A. brasiliensis ATCC 16404); (D) itraconazole (*** $P<0.00$ I compared to F. solani ATCC 3636 strains; @@@P $<0.00$ I compared to F. solani ATCC 2667 I strains; ${ }^{P}<0.05$ compared to A. fumigatus ATCC 90906 strains); and (E) fluconazole. (F) Representative photographs showing the zone of inhibition of gelatin (upper panel) and amphotericin B-loaded gelatin (lower panels) fiber mats.

Note: The absence of bars in the graph indicates no inhibition against the particular strain.

Abbreviation: ATCC, American Tissue Culture Collection.

The release profile was similar for all the antifungals, although the amount of released drugs was different. The sustained release of polyene antifungals from fiber mats indicated significant drug-gelatin interactions. ${ }^{38}$ At the end of 3 days, the amount of amphotericin $\mathrm{B}$ and natamycin released in the medium was $9.5 \mu \mathrm{g} / \mathrm{mL}$ and $8.5 \mathrm{mg} / \mathrm{mL}$, respectively. The amount of terbinafine released was $7.3 \mu \mathrm{g} / \mathrm{mL}$. Of all the antifungals, fluconazole was released in maximum amounts $(14 \mu \mathrm{g} / \mathrm{mL})$. To understand the loss of activity for other antifungals when incorporated into the gelatin matrix, the MIC of all the antifungals alone against five different C. albicans strains was determined. Table 1 compares the determined MIC of various antifungal agents. Amphotericin B displayed the lowest MIC followed by natamycin against all the $C$. albicans strains examined. Interestingly, four C. albicans strains showed resistance to fluconazole as the MIC was $>64 \mu \mathrm{g} / \mathrm{mL}$. A good correlation was observed between the amounts of antifungals released and MIC values of free antifungals against particular strains of C. albicans. Thus, poor antifungal properties and release characteristics 


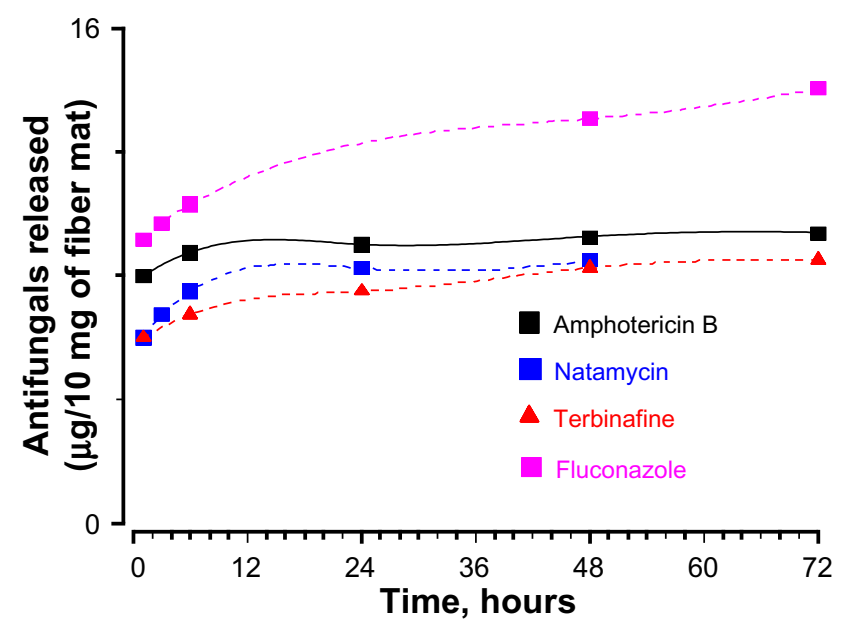

Figure 5 Cumulative release profile of antifungals form gelatin fiber mats.

are responsible for apparent loss of activity of azoles and terbinafine upon incorporation into the gelatin matrix.

\section{Kinetics of candidacidal action of polyene-loaded fiber mats}

Since both the polyene-loaded fiber mats displayed generally good inhibitory activity, the remaining experiments were performed with amphotericin B- and natamycin-loaded fiber mats. After incubation for 52 hours, both the polyene-loaded fiber mats resulted in considerable loss of viability ( $\geq 2 \log$ reduction in viability) of C. albicans (Figure 6A), suggesting potent candidacidal action. To gain further insights, the kinetics of candidacidal action after exposing $C$. albicans $\left(1.5 \times 10^{5} \mathrm{CFU} / \mathrm{mL}\right)$ to amphotericin B- or natamycin-loaded fiber mats was also investigated (Figure 6B). At various time intervals, the supernatant was diluted $\left(10^{2}\right.$ or $10^{3}$ fold $)$, plated on Sabouraud dextrose agar, and the number of yeast colonies was counted after 48 hours. For both the polyene antifungal-loaded fiber mats, a steep decrease in the viability of $C$. albicans was observed during early incubation time. After 24 hours of incubation, amphotericin B-loaded fiber mats caused $1.6 \log _{10} \mathrm{CFU} / \mathrm{mL}$ decrease (97.3\% killing), whereas natamycin caused $1.2 \log \log _{10} \mathrm{CFU} / \mathrm{mL}$ decrease ( $93 \%$ killing) in the viability of C. albicans.
The changes in morphology of C. albicans incubated with polyene-loaded fiber mats were investigated by SEM. C. albicans incubated with gelatin alone had smooth and intact surfaces (Figure 7A). However, cells treated with the polyene antifungals had extensive surface deformation and leakage of some intracellular components (Figure 7B and C). The damage caused by antifungals was more noticeable in the amphotericin B-loaded fiber mats than in natamycin-loaded fiber mats. These results confirm that the polyene-loaded fiber mats retained the antifungal activity even in the presence of gelatin.

\section{Interaction of gelatin with antifungals}

Since the fiber diameter of gelatin mats was doubled in the presence of polyene-loaded antifungals, the interaction between antifungals and gelatin was investigated using spectroscopy and rheometry. First, the changes in secondary structure of gelatin were monitored by $\mathrm{CD}$ spectropolarimetry. Figure 8A depicts the changes in the secondary structure of the gelatin solution and gelatin upon incubation with $0.25 \mathrm{wt} \%$ amphotericin B and natamycin. The CD spectra of gelatin showed a strong negative trough around $197 \mathrm{~nm}$ and no broad positive band in the $\mathrm{n}-\pi^{*}$ region (Figure 8A). However, gelatin incubated with natamycin and amphotericin $\mathrm{B}$ displayed a strong maximum in the $n-\pi *$ region (Figure $8 \mathrm{~A}$ ). The positive maximum was more pronounced for natamycin compared to amphotericin $\mathrm{B}$. The presence of a positive band around $225 \mathrm{~nm}$ is a hallmark for triple helical structure associated with collagen. ${ }^{39,40}$ Using the equation reported by Fujitsu et al, the relative helical content $\left([\mathrm{C}]_{\mathrm{rel}}\right)$ of gelatin in the presence of polyenes was estimated ${ }^{41}$ Positive or negative $(\mathrm{C})_{\text {rel }}$ indicates increased or decreased triple helical content, respectively. $(\mathrm{C})_{\text {rel }}$ at $10^{\circ} \mathrm{C}$ for amphotericin B- and natamycin-loaded gelatin was 0.8 and 2.4, respectively, indicating greater stability of triple helix structure in the presence of natamycin.

To further confirm these observations, the variable temperature $\mathrm{CD}$ was recorded by monitoring the change in ellipticity at $225 \mathrm{~nm}$. Thermal transition has been used to investigate the helix-coil transition in collagen or collagen

Table I Minimum inhibitory concentration of antifungals against various strains of Candida albicans

\begin{tabular}{|c|c|c|c|c|c|}
\hline \multirow[t]{2}{*}{ Antifungals } & \multicolumn{5}{|c|}{ Minimum inhibitory concentration in $\mu \mathrm{g} / \mathrm{mL}$ against } \\
\hline & $\begin{array}{l}\text { C. albicans } \\
\text { ATCC I023I }\end{array}$ & $\begin{array}{l}\text { C. albicans } \\
\text { ATCC } 209 \text { I }\end{array}$ & $\begin{array}{l}\text { C. albicans } \\
\text { ATCC } 24433\end{array}$ & $\begin{array}{l}\text { C. albicans } \\
\text { DF } 2672 R\end{array}$ & $\begin{array}{l}\text { C. albicans } \\
\text { DF 1976R }\end{array}$ \\
\hline AmpB & 1.25 & 1.25 & 1.25 & 1.25 & 1.25 \\
\hline Nata & 10 & 5 & 10 & 10 & 10 \\
\hline Terb & 16 & 64 & $>64$ & 8 & 16 \\
\hline Fluco & $>64$ & $>64$ & 16 & $>128$ & $>128$ \\
\hline
\end{tabular}

Abbreviations: AmpB, amphotericin B;ATCC,American Type Culture Collection; C. albicans, Candida albicans; Fluco, fluconazole; Nata, natamycin; Terb, terbinafine chloride. 
A

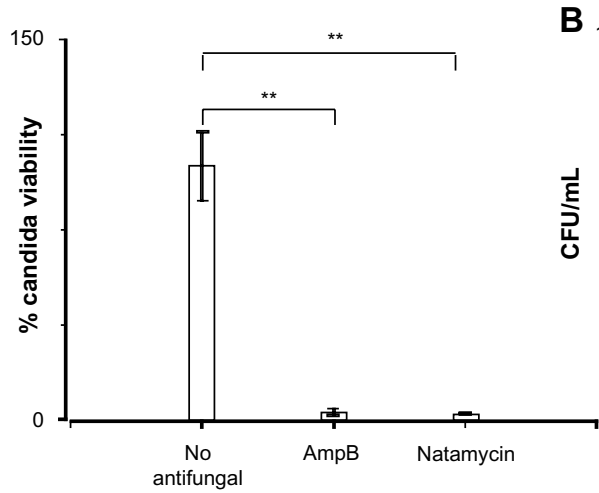

B

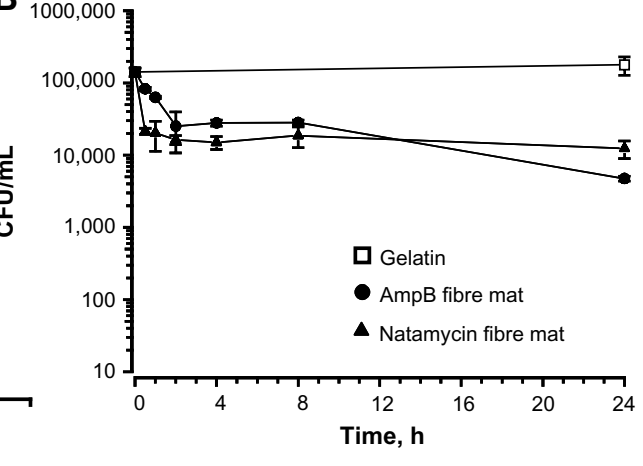

Figure 6 (A) Candidacidal properties of the fiber mats loaded with amphotericin B and natamycin. (B) Kinetics of candidacidal action of amphotericin B and natamycinloaded fiber mats.

Notes: There was a significant decrease in the viability of Candida albicans exposed to antifungal-loaded fiber mats; $* * P<0.01$.

Abbreviation: AmpB, amphotericin B.

mimetic peptides. ${ }^{42,43}$ For gelatin without antifungals, a linear change in ellipticity $\left([\theta]_{225}\right)$ with temperature was observed suggesting denatured conformation and lack of a triple helix structure (Figure 8B). However, gelatin incubated with natamycin and amphotericin B displayed a nonlinear change in ellipticity, indicating clear helix-coil transition (Figure 8B and $\mathrm{C}$ ). As was observed before, natamycin promoted the helical conformation more significantly than amphotericin B. Sugars and polyols can protect the native structure and promote helix-coil transition of gelatin by crosslinking through
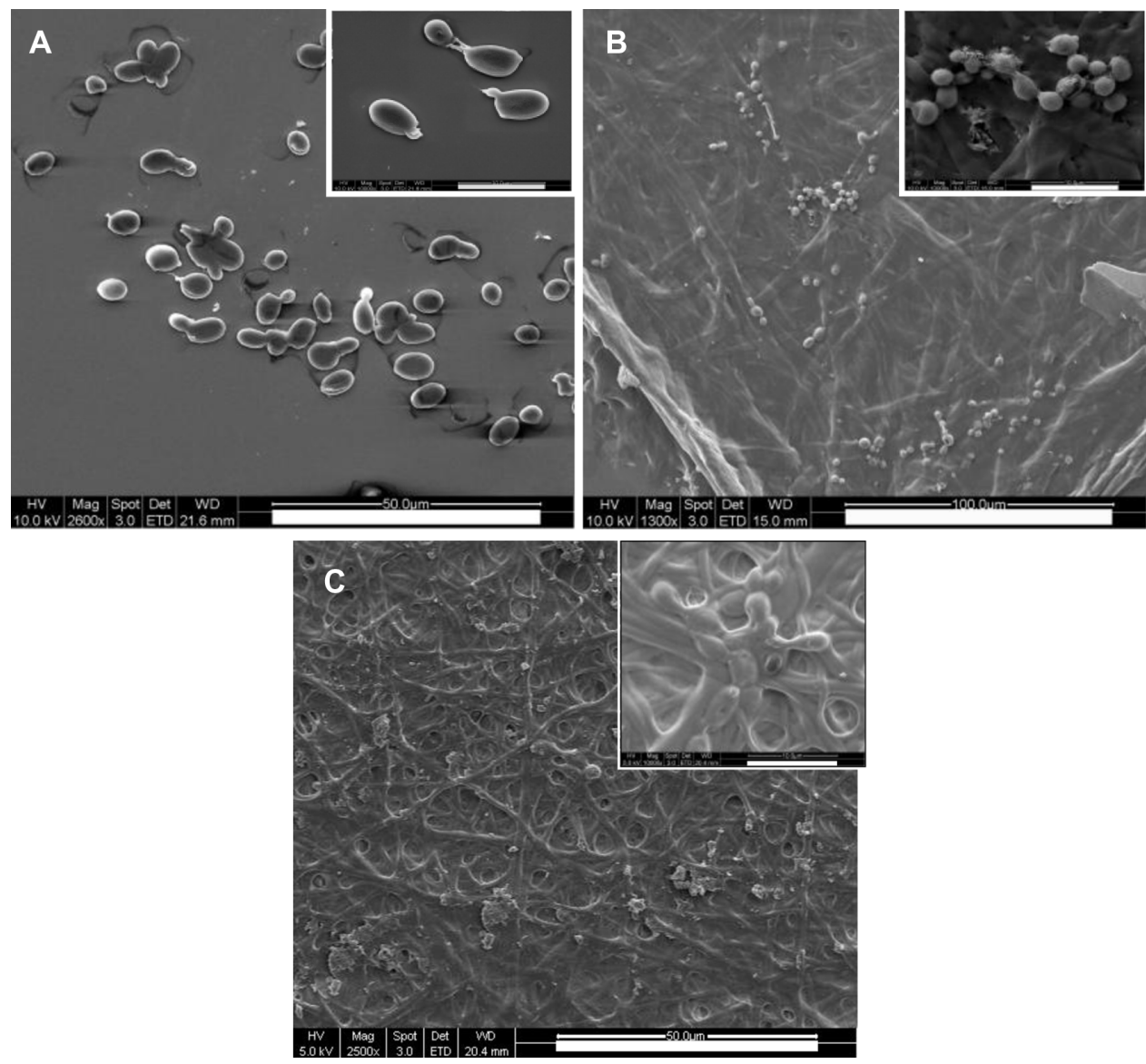

Figure 7 Morphology of Candida albicans grown on polyene-loaded fiber mats. Scanning electron micrographs of $C$. albicans treated with (A) gelatin fiber mats without antifungals; (B) amphotericin B-loaded fiber mats; and (C) natamycin-loaded fiber mats.

Note: Inset scale bar $=10 \mu \mathrm{m}$. 

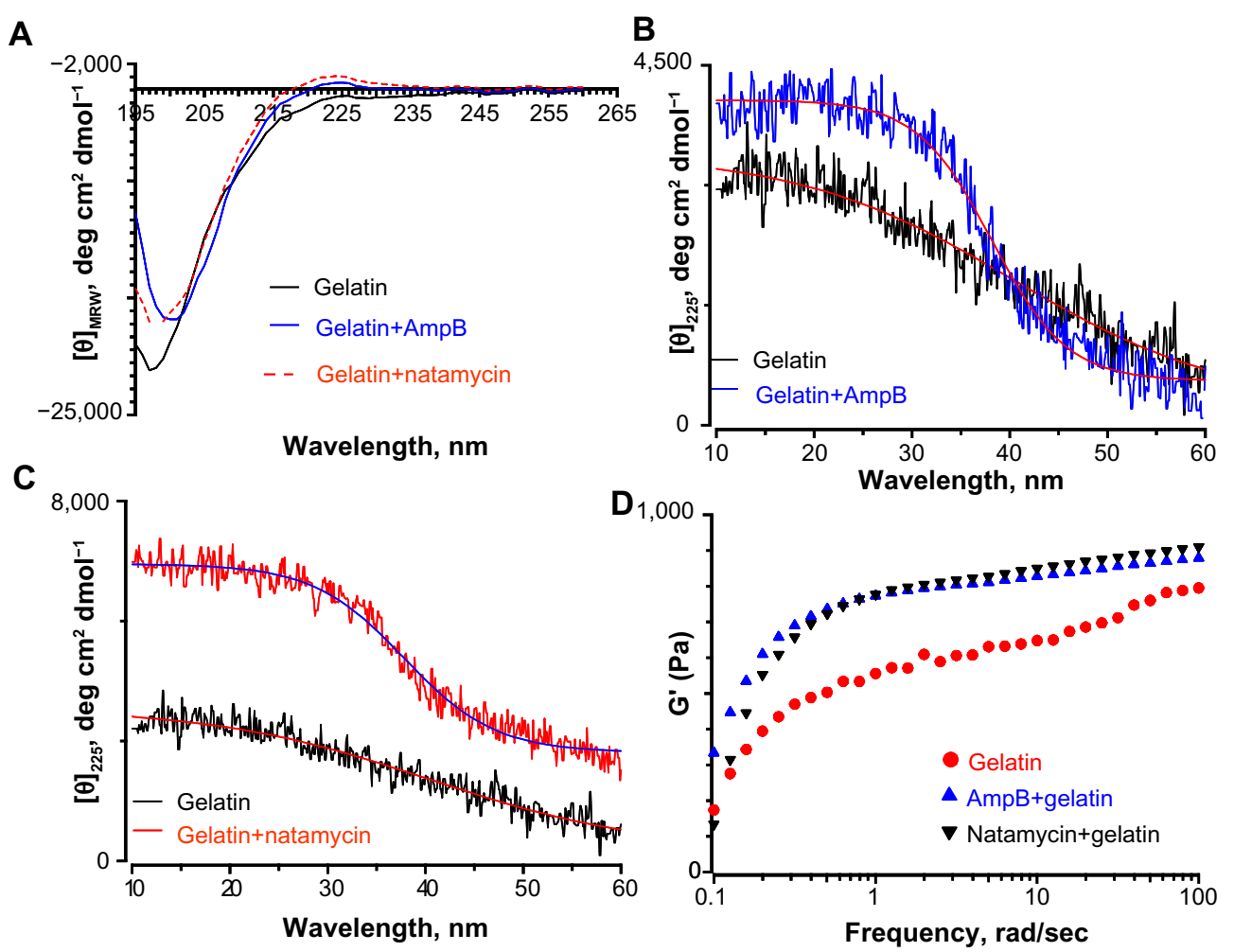

Figure 8 Interaction between polyene antifungals and gelatin probed by circular dichroism spectropolarimetry and rheological studies. (A) Far ultraviolet circular dichroism spectropolarimetry of gelatin and gelatin incubated with polyene antifungals at $30^{\circ} \mathrm{C}$. Thermal denaturation of gelatin in the presence of $(\mathbf{B})$ amphotericin $\mathbf{B}$ and $(\mathbf{C})$ natamycin. The concentration of antifungals was 0.25 wt\% with respect to gelatin. (D) Frequency sweep of storage modulus ( $\left.\mathrm{G}^{\prime}\right)$ of gelatin and antifungal-loaded gelatin. Abbreviation: AmpB, amphotericin B.

hydrogen bonding. ${ }^{41,44}$ The results suggest that the greater stabilization of gelatin by the two polyenes is attributed to a significantly higher number of hydroxyl groups present (ten in amphotericin B and five in natamycin). However, at this stage, it is difficult to conceive the increased triple helical propensity in the presence of natamycin over amphotericin B, though the latter has a higher number of $-\mathrm{OH}$ groups. In the presence of terbinafine, a small increase in helical propensity was observed, whereas fluconazole had no significant influence on the secondary structure of gelatin (Figure S8). The estimated $(\mathrm{C})_{\text {rel }}$ was 0.78 in the presence of terbinafine and 0.5 for fluconazole-loaded gelatin, thus confirming a weak interaction between fluconazole and gelatin.

Rheological studies of the drug-loaded gelatin solutions were carried out to determine if the interaction of antifungals modified the physical properties of the gels. Macroscopically, a nonflowable gel was obtained with or without drug. This material did not flow even when the sample vial was inverted. A solid-like behavior was observed with $\mathrm{G}^{\prime}$ greater than $\mathrm{G}^{\prime \prime}$ under these conditions, and both the moduli were almost independent of frequency at higher frequency ranges (Figures $8 \mathrm{D}$ and S9). The frequency-independent value of $\mathrm{G}^{\prime}$ for gelatin solution without any antifungals was $767 \pm 27 \mathrm{~Pa}$. The $\mathrm{G}^{\prime}$ value was increased to $851 \pm 7 \mathrm{~Pa}$ and $848 \pm 9 \mathrm{~Pa}$ in the presence of amphotericin B and natamycin, respectively. Combined with CD studies, it is likely that the increased association between the gelatin chains brought about by the supramolecular interactions between the polyenes and the protein chains through hydrogen bonding results in increased storage modulus and increased diameter of the fibers. This interesting phenomenon certainly warrants further investigation and suggests that rheology, along with spectrometry methods, could provide valuable information on drug-matrix interactions.

\section{Gelatin stabilizes the monomeric forms of amphotericin B}

It has been shown that the toxicity of amphotericin B for mammalian cells depends on the level of aggregation. ${ }^{45,46} \mathrm{In}$ aqueous solutions, amphotericin B exists in an aggregated form containing about 2,000 molecules, whereas in DMSO the monomeric form is predominant below $5 \mathrm{mM} .{ }^{47}$ The characteristic absorption bands in UV and CD spectroscopic methods can differentiate between the different states of aggregation. Therefore, the UV absorption spectra of amphotericin B under various conditions were recorded. In DMSO, amphotericin B displayed four clear characteristic absorption bands around 
350, 368, 388, and $412 \mathrm{~nm}$ (Figure 9A). In buffer, the intensity of well-defined absorption bands decreased and a broad band around $340 \mathrm{~nm}$ was observed. However, the presence of gelatin lowers the extent of aggregation of amphotericin B and all four major peaks were visible (Figure 9A). The band around $412 \mathrm{~nm}$ is a measure of monomeric form of amphotericin $\mathrm{B}$, whereas the band at $346 \mathrm{~nm}$ is assigned to an aggregated form. ${ }^{48}$ The ratio of two peak intensities at $346 \mathrm{~nm}$ and $412 \mathrm{~nm}$
A

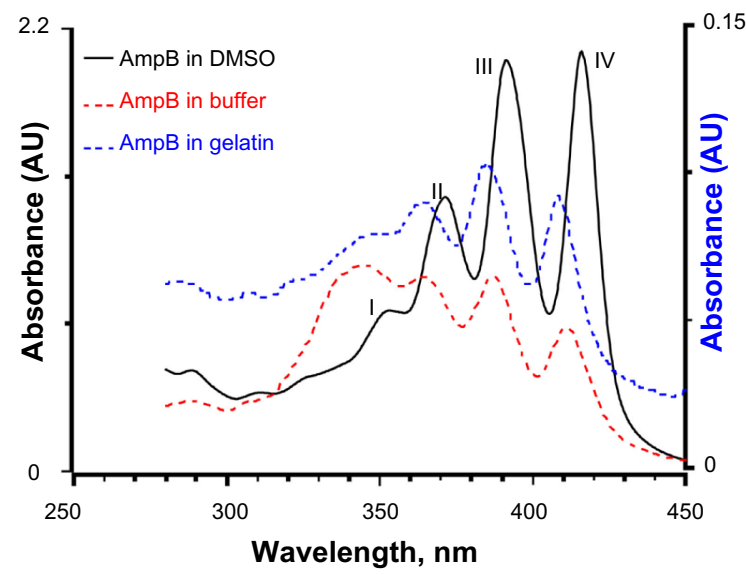

C 2500

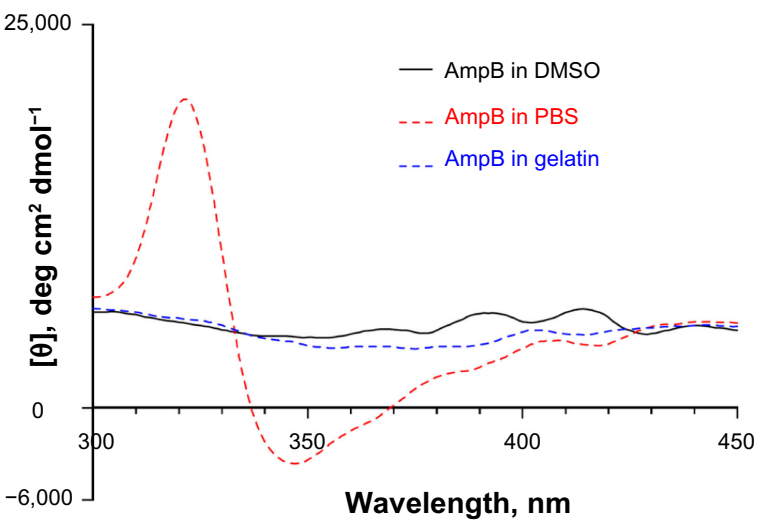

E

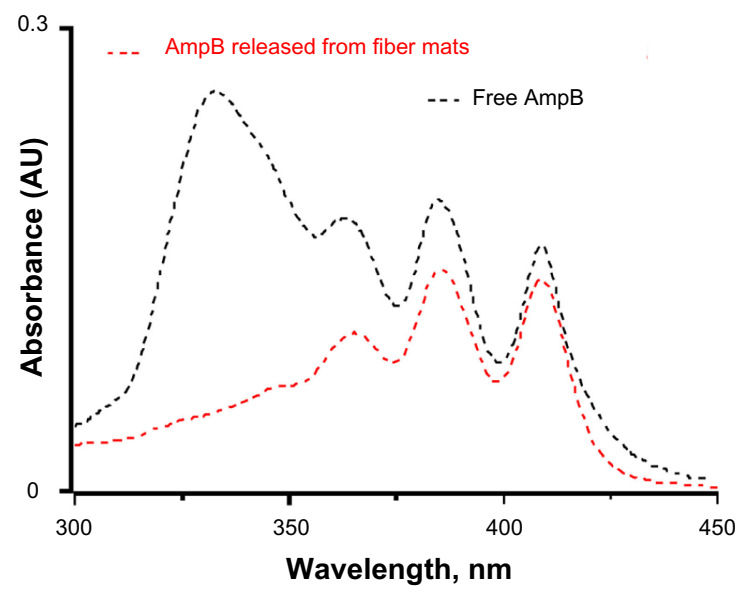

B
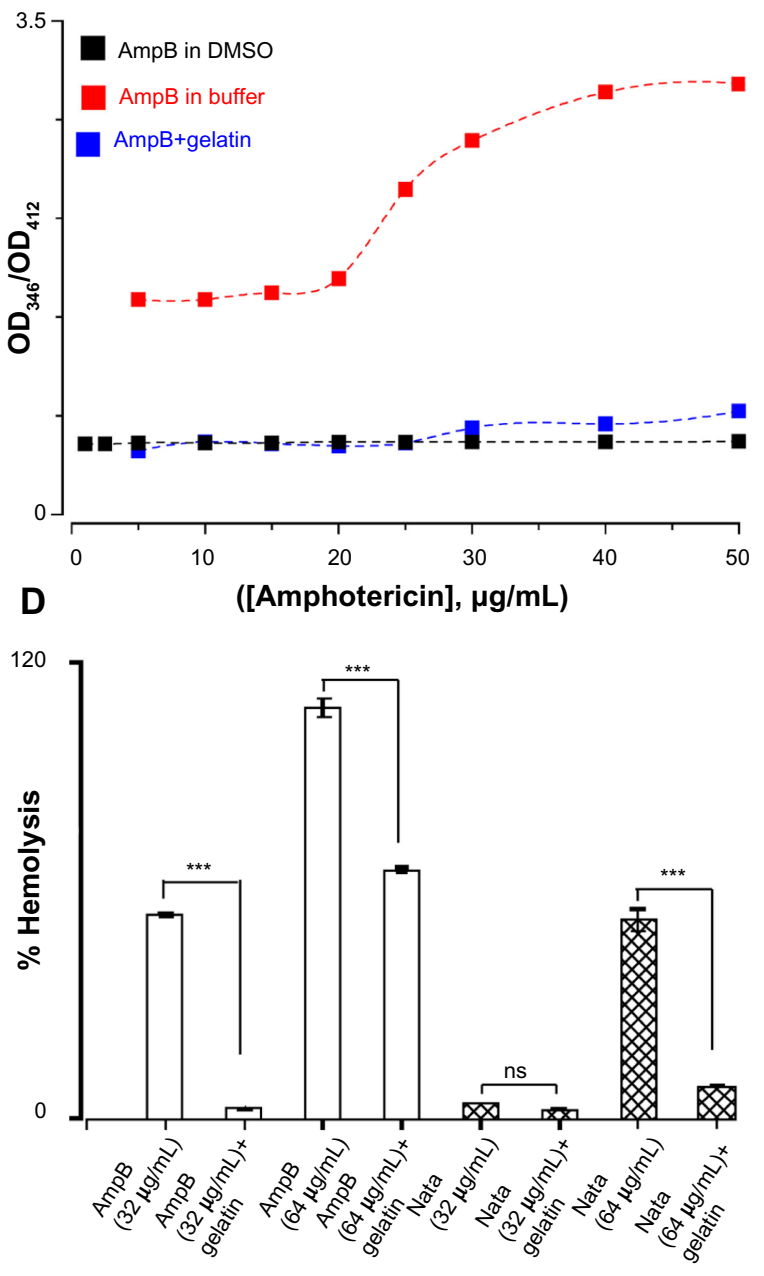

Figure 9 (A) Ultraviolet absorption spectra of amphotericin B in three different media. The four peaks observed in the monomeric amphotericin B are labelled. The concentration of amphotericin used was $50 \mu \mathrm{g} / \mathrm{mL}$. The $\mathrm{Y}$-axis for the amphotericin B in gelatin is represented separately. (B) The relative aggregation state of amphotericin $B$ as measured by the intensity ratio of peak I and peak IV of the absorption spectra in three different media. (C) Circular dichroism spectrum of amphotericin B in three different media. Note the bisignate nature of amphotericin B in phosphate buffered saline indicating a complex structure. (D) Hemolytic activity of amphotericin B and natamycin in the presence/absence of gelatin. For all these studies, the antifungal:gelatin ratio was 0.25 wt\%. (E) Ultraviolet absorption spectra of amphotericin B released from the gelatin matrix. For a comparison, the absorption spectra of free amphotericin $B(10 \mu \mathrm{g} / \mathrm{mL})$ in buffer is also shown.

Note: $* * * P<0.001$.

Abbreviations: AmpB, amphotericin B; DMSO, dimethyl sulfate; PBS, phosphate buffered saline; AU, arbitrary unit. 
determines the extent of aggregation of amphotericin B. In DMSO, a peak ratio of 0.5 was observed as the concentration of amphotericin B was varied from 1-50 $\mu \mathrm{g} / \mathrm{mL}$, indicating the presence of monomeric forms (Figure 9B). In buffer, the peak ratios were 1.5 at lower and 3.1 at higher concentrations of amphotericin B. However, in the presence of gelatin, the peak ratio was 0.47 below $20 \mu \mathrm{g} / \mathrm{mL}$ and increased to 0.73 at $50 \mu \mathrm{g} / \mathrm{mL}$ of amphotericin $\mathrm{B}$, thus confirming that gelatin prevents the aggregation of amphotericin B in aqueous solution (Figure 9B).

To confirm these results further, the CD spectra of amphotericin B in DMSO, PBS buffer, and in the presence of gelatin were also monitored (Figure 9C). Amphotericin B displayed three positive bands at 412, 390, and $364 \mathrm{~nm}$ which are characteristic of conjugated systems ${ }^{47}$ In PBS, a bisignate shape was observed suggesting supramolecular structure of amphotericin B in aqueous solutions. A strong negative trough around $346 \mathrm{~nm}$ and an intense positive peak around $320 \mathrm{~nm}$ were observed in PBS. However, in the presence of gelatin, the spectrum was closer to the one observed in DMSO and no intense peak around $320 \mathrm{~nm}$ was observed. ${ }^{47}$ Based on UV absorption and CD studies, it was concluded that the amphotericin B interacts with gelatin in the monomeric/oligomeric form and in turn promotes the triple helical conformation.

\section{Hemolytic activity of antifungal- loaded gelatin}

Since, in its aggregated form, amphotericin B forms ion channels in cholesterol-containing membrane, the hemolytic activity of polyene drugs in the presence of gelatin was assessed. ${ }^{49,50}$ As shown in Figure 9D, the interaction of amphotericin B with gelatin resulted in a significant decrease in the hemolysis of rabbit erythrocytes compared to free drug at $32 \mu \mathrm{g} / \mathrm{mL}$ and $64 \mu \mathrm{g} / \mathrm{mL}$. Similarly, interaction of natamycin with gelatin also resulted in decreased hemolysis at $64 \mu \mathrm{g} / \mathrm{mL}$. These results suggest that the interaction between polyene drugs and gelatin is complementary, leading to increased triple helical propensity of gelatin and stabilization of amphotericin B in monomeric/oligomeric forms which otherwise tend to form higher order aggregates in aqueous buffers. The stabilization of monomeric/oligomeric forms of amphotericin B accounts for the decreased hemolytic activity. Furthermore, controlled release studies from crosslinked gelatin fiber mats further confirmed that the released amphotericin B displayed four distinct peaks that are characteristic of monomeric forms (Figure 9E).

\section{Cytotoxicity of antifungal-loaded gelatin fiber mats}

The biocompatibility of polyene-loaded antifungals was evaluated by monitoring the cell viability of human corneal fibroblasts and human sclera fibroblasts primary cells. For a comparison we have also determined the cytotoxicity of free polyenes with human corneal fibroblast cells (Figure 10A). At $10 \mu \mathrm{g} / \mathrm{mL}$, both natamycin and amphotericin B did not show significant cytotoxicity to the human corneal fibroblasts. Gelatin gels without glutaraldehyde crosslinking also did not show any toxicity. However, 24-hour glutaraldehyde crosslinked gelatin fiber mats displayed significant toxicity. Natamycin-loaded fiber mats displayed better compatibility with the human corneal fibroblasts $(\sim 90 \%$ viable cells) compared to amphotericin B-loaded fiber mats ( $\sim 67 \%$ viable cells). However, for primary human sclera fibroblasts, both the antifungal-loaded fiber mats were equally less toxic ( $70 \%$ viable cells; Figure $10 \mathrm{~B})$. These results indicate excellent biocompatibility of fiber mats with human fibroblasts.
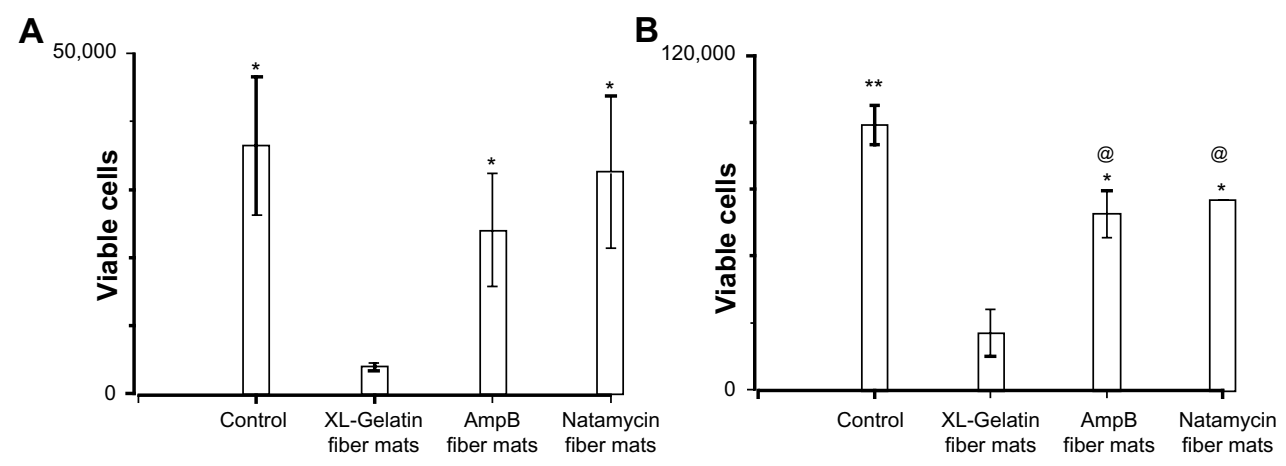

Figure 10 Cytotoxicity of polyene-loaded antifungal fiber mats against (A) human corneal and (B) human sclera fibroblasts. XL-gelatin fiber mat is the gelatin crosslinked with glutaraldehyde for 24 hours. For amphotericin B- and natamycin-loaded fiber mats the crosslinking time was 3 hours.

Notes: $* \mathrm{P}<0.05$ compared to $\mathrm{XL}$-gelatin fiber mats; $* * \mathrm{P}<0.01$ compared to $\mathrm{XL}$-gelatin fiber mats; $@ \mathrm{P}<0.05$ compared to control.

Abbreviation: AmpB, amphotericin B. 


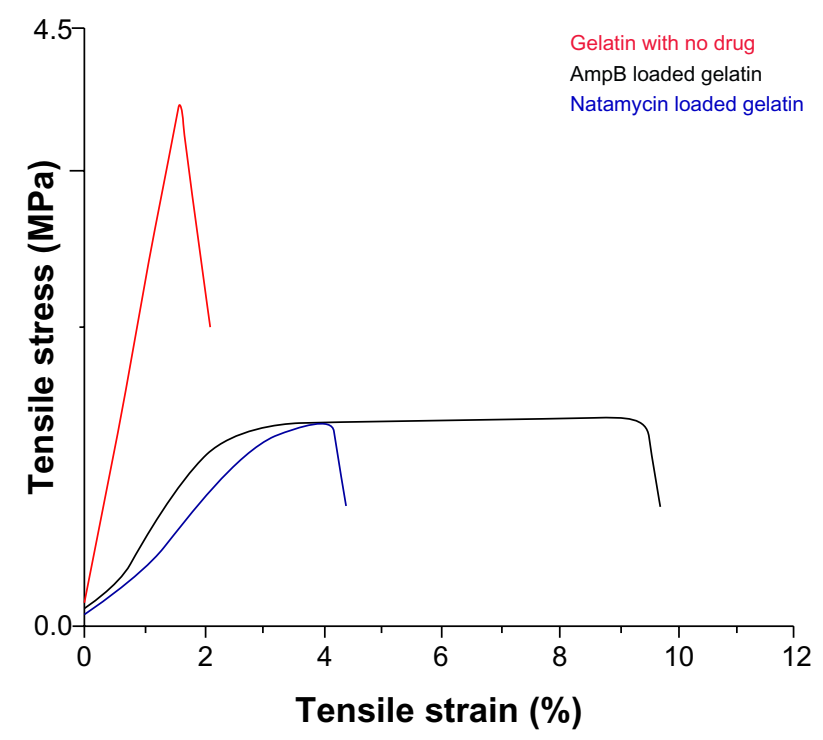

Figure II Mechanical properties of glutaraldehyde vapor crosslinked gelatin nanofibers with and without drug loading. The gelatin fiber mat was crosslinked with glutaraldehyde for $\mathbf{4 8}$ hours. For amphotericin B- and natamycin-loaded gelatin fiber mats 3-hour glutaraldehyde crosslinking was used.

Abbreviation: $\mathrm{AmpB}$, amphotericin $\mathrm{B}$.

\section{Mechanical properties of polyene-loaded fiber mats}

The mechanical properties of polyene-loaded fiber mats were evaluated (Figure 11) and compared with uncrosslinked and crosslinked gelatin mats (Table 2). Mechanical strength measurements showed a slight increase in Young's modulus of antifungal-loaded gelatin compared to uncrosslinked gelatin. For natamycin-loaded fiber mats, the ultimate tensile stress and the ultimate tensile strain decreased compared to uncrosslinked gelatin fiber mats. However, amphotericin B-loaded fiber mats had improved tensile strain, although a slight decrease in the ultimate tensile stress was observed compared to the uncrosslinked gelatin fiber mats. When compared to crosslinked gelatin fibers, the antifungal-loaded

Table 2 Mechanical properties of polyene-loaded fiber mats

\begin{tabular}{|c|c|c|c|}
\hline Fiber mats & $\begin{array}{l}\text { Young's } \\
\text { modulus } \\
(\mathrm{MPa})\end{array}$ & $\begin{array}{l}\text { Ultimate } \\
\text { tensile stress } \\
(\mathrm{MPa})\end{array}$ & $\begin{array}{l}\text { Ultimate } \\
\text { tensile } \\
\text { strain (\%) }\end{array}$ \\
\hline Gelatin (not crosslinked) & 25.7 & 1.9 & 5.5 \\
\hline $\begin{array}{l}\text { Gelatin (crosslinked } \\
\text { with glutaraldehyde for } \\
24 \text { hours) }\end{array}$ & 92.4 & 2.9 & 2.9 \\
\hline $\begin{array}{l}\text { Amphotericin B-gelatin } \\
\text { (crosslinked with } \\
\text { glutaraldehyde for } 3 \text { hours) }\end{array}$ & $31.5 \pm 0.9$ & $1.6 \pm 0.3$ & $9.6 \pm 1.4$ \\
\hline $\begin{array}{l}\text { Natamycin-gelatin } \\
\text { (crosslinked with } \\
\text { glutaraldehyde for } 3 \text { hours) }\end{array}$ & $29.3 \pm 0.9$ & $1.5 \pm 0.2$ & $3.7 \pm 1.1$ \\
\hline
\end{tabular}

fibers had improved elasticity, an important requirement for wound dressing. ${ }^{33}$

\section{Conclusion}

In this work, the in vitro efficacy and toxicity of various US Food and Drug Administration-approved antifungal-loaded electrospun fiber mats were compared. Of the three classes of antifungals that were compared, the polyene-loaded fiber mats displayed excellent antifungal activities against a wide range of pathogenic yeasts and fungi, including filamentous Aspergillus spp. and Fusarium spp. The polyene-loaded fiber mats resulted in a significant reduction in the viability of C. albicans as well as rapid candidacidal action. Conversely, the polyene-loaded fiber mats did not decrease the viability corneal and sclera fibroblasts, suggesting excellent selectivity and biocompatibility. Using various spectroscopic and rheological measurements, it was demonstrated that polyene antifungals interact with a gelatin matrix, which decreases the hemolytic activity without significantly altering the antifungal activity. These characteristics of polyene-loaded fiber mats would be suitable for the management of superficial fungal infections of the skin and the cornea. The poor release characteristics and weak antifungal properties are responsible for the complete loss of activity of fluconazoleloaded fiber mats. Antimicrobial nanofibers have been realized as promising scaffolds in many areas such as controlled drug delivery, wound dressing, tissue engineering, stem cell regeneration and differentiation, and food packaging. ${ }^{38,51-53}$ Hydrogel systems, in particular, are potentially advantageous for these applications. ${ }^{54-58}$ To the best of the authors' knowledge, this is the first report that compares the in vitro efficacy of various antifungal-loaded fiber mats and examines their physicochemical interactions with gelatin.

\section{Acknowledgments}

This work is supported by TCR Grant R618/41/2008 (RWB), R1054/69/2013 (RL), the NRF-Technion R-398-001-065592 (SR), A*STAR-BEP R-265-000-437-305 (SR), and Department of Mechanical Engineering, National University of Singapore, Singapore.

\section{Disclosure}

The authors report no conflicts of interest in this work.

\section{References}

1. Brown GD, Denning DW, Levitz SM. Tackling human fungal infections. Science. 2012;336(6082):647.

2. Fisher MC, Henk DA, Briggs CJ, et al. Emerging fungal threats to animal, plant, and ecosystem health. Nature. 2012;484(7393):186-194. 
3. Brown GD, Denning DW, Gow NA, Levitz SM, Netea MG, White TC. Hidden killers: human fungal infections. Sci Transl Med. 2012;4(165): 165 rv13.

4. Havlickova B, Czaika VA, Friedrich M. Epidemiological trends in skin mycoses worldwide. Mycoses. 2008;51(Suppl 4):2-15.

5. Lai CC, Tan CK, Huang YT, Shao PL, Hsueh PR. Current challenges in the management of invasive fungal infections. J Infect Chemother. 2008;14(2):77-85.

6. Lupi O, Tyring SK, McGinnis MR. Tropical dermatology: fungal tropical diseases. J Am Acad Dermatol. 2005;53(6):931-951.

7. Ellis M. Invasive fungal infections: evolving challenges for diagnosis and therapeutics. Mol Immunol. 2002;38(12-13):947-957.

8. Sanglard D, Ischer F, Parkinson T, Falconer D, Bille J. Candida albicans mutations in the ergosterol biosynthetic pathway and resistance to several antifungal agents. Antimicrob Agents Chemother. 2003;47(8): 2404-2412.

9. Perlin DS. Antifungal drug resistance: do molecular methods provide a way forward? Curr Opin Infect Dis. 2009;22(6):568-573.

10. Masia CM, Gutierrez RF. Antifungal drug resistance to azoles and polyenes. Lancet Infect Dis. 2002;2(9):550-563.

11. Butts A, Krysan DJ. Antifungal drug discovery: something old and something new. PLoS Pathog. 2012;8(9):e1002870.

12. Ostrosky-Zeichner L, Casadevall A, Galgiani JN, Odds FC, Rex JH. An insight into the antifungal pipeline: selected new molecules and beyond. Nat Rev Drug Discov. 2010;9(9):719-727.

13. Hsu LY, Wijaya L, Shu-Ting Ng E, Gotuzzo E. Tropical fungal infections. Infect Dis Clin North Am. 2012;26(2):497-512.

14. Kaur IP, Kakkar S. Topical delivery of antifungal agents. Expert Opin Drug Deliv. 2010;7(11):1303-1327.

15. Zumbuehl A, Ferreira L, Kuhn D, et al. Antifungal hydrogels. Proc Natl Acad Sci U S A. 2007;104(32):12994-12998.

16. Zhang L, Pornpattananangku D, Hu CM, Huang CM. Development of nanoparticles for antimicrobial drug delivery. Curr Med Chem. 2010;17(6):585-594.

17. Zarif L, Graybill JR, Perlin D, Najvar L, Bocanegra R, Mannino RJ. Antifungal activity of amphotericin $\mathrm{B}$ cochleates against Candida albicans infection in a mouse model. Antimicrob Agents Chemother. 2000;44(6):1463-1469.

18. Gupta M, Goyal AK, Paliwal SR, et al. Development and characterization of effective topical liposomal system for localized treatment of cutaneous candidiasis. J Liposome Res. 2010;20(4):341-350.

19. Bhalaria MK, Naik S, Misra AN. Ethosomes: a novel system for antifungal drugs in the treatment of topical fungal disease. Indian $J$ Exp Biol. 2009;47(5):368-375.

20. Ramos G, Cuenca-Estrella M, Monzon A, Rodriguez-Tudela JL. In-vitro comparative activity of UR-9825, itraconazole and fluconazole against clinical isolates of Candida spp. J Antimicrob Chemother. 1999;44(2): 283-286.

21. Prabhu P, Patravale V, Joshi M. Nanocarriers for effective topical delivery of anti-infectives. Curr Nanosci. 2012;8(4):491-503.

22. Teo WE, Ramakrishna S. A review on electrospinning design and nanofiber assemblies. Nanotechnology. 2006;17(14):R89-R106.

23. Greiner A, Wendorff JH. Electrospinning: a fascinating method for the preparation of ultrathin fibers. Angew Chem Int Ed Engl. 2007;46(30):5670-5703.

24. Murugan R, Ramakrishna S. Design strategies of tissue engineering scaffolds with controlled fiber orientation. Tissue Eng. 2007;13(8): 1845-1866.

25. Katti DS, Robinson KW, Ko FK, Laurencin CT. Bioresorbable nanofiber-based systems for wound healing and drug delivery: optimization of fabrication parameters. J Biomed Mater Res B Appl Biomater. 2004;70(2):286-296.

26. Zahedi P, Rezaeian I, Ranaei-Siadat SO, Jafari SH, Supaphol P. A review on wound dressings with an emphasis on electrospun nanofibrous polymeric bandages. Polym Adv Technol. 2010;21(2):77-95.

27. Malmsten M. Antimicrobial and antiviral hydrogels. Soft Matter. 2011;7(19):8725-8736.
28. Schiffman JD, Elimelech M. Antibacterial activity of electrospun polymer mats with incorporated narrow diameter single-walled carbon nanotubes. ACS Appl Mater Interfaces. 2011;3(2):462-468.

29. Tan K, Obendorf SK. Fabrication and evaluation of electrospun nanofibrous antimicrobial nylon 6 membranes. J Memb Sci. 2007;305(1-2):287-298.

30. Chen L, Bromberg L, Hatton TA, Rutledge GC. Electrospun cellulose acetate fibers containing chlorhexidine as a bactericide. Polymer. 2008;49(5):1266-1275.

31. Taepaiboon P, Rungsardthong U, Supaphol P. Vitamin-loaded electrospun cellulose acetate nanofiber mats as transdermal and dermal therapeutic agents of vitamin A acid and vitamin E. Eur J Pharm Biopharm. 2007;67(2):387-397.

32. Ignatova M, Stoilova O, Manolova N, Markova N, Rashkov I. Electrospun mats from styrene/maleic anhydride copolymers: modification with amines and assessment of antimicrobial activity. Macromol Biosci. 2010;10(8):944-954.

33. Bshena O, Heunis TD, Dicks LM, Klumperman B. Antimicrobial fibers: therapeutic possibilities and recent advances. Future Med Chem. 2011;3(14):1821-1847.

34. Lynn AK, Yannas IV, Bonfield W. Antigenicity and immunogenicity of collagen. J Biomed Mater Res B Appl Biomater. 2004;71(2): 343-354.

35. Liu X, Smith LA, Hu J, Ma PX. Biomimetic nanofibrous gelatin/ apatite composite scaffolds for bone tissue engineering. Biomaterials. 2009;30(12):2252-2258.

36. Choktaweesap N, Arayanarakul K, Aht-ong D, Meechaisue C, Supaphol P. Electrospun gelatin fibers: effect of solvent system on morphology of and fiber diameters. Polym J. 2007;39:622-631.

37. Oren Z, Shai Y. Selective lysis of bacteria but not mammalian cells by diastereomers of melittin: structure-function study. Biochemistry. 1997;36(7):1826-1835.

38. Ignatova M, Rashkov I, Manolova N. Drug-loaded electrospun materials in wound-dressing applications and in local cancer treatment. Expert Opin Drug Deliv. 2013;10(4):469-483.

39. Xu P, Huang J, Cebe P, Kaplan DL. Osteogenesis imperfecta collagen-like peptides: self-assembly and mineralization on surfaces. Biomacromolecules. 2008;9(6):1551-1557.

40. Beck K, Chan VC, Shenoy N, Kirkpatrick A, Ramshaw JA, Brodsky B. Destabilization of osteogenesis imperfecta collagen-like model peptides correlates with the identity of the residue replacing glycine. Proc Natl Acad Sci US A. 2000;97(8):4273-4278.

41. Fujitsu M, Hattori M, Tamura T. Effects of hydroxy compounds on gel formation of gelatin. Colloid Polym Sci. 1997;275(1):67-72.

42. Xu K, Nowak I, Kirchner M, Xu Y. Recombinant collagen studies link the severe conformational changes induced by osteogenesis imperfecta mutations to the disruption of a set of interchain salt bridges. $J$ Biol Chem. 2008;283(49):34337-34344.

43. Li Y, Foss CA, Summerfield DD, et al. Targeting collagen strands by photo-triggered triple-helix hybridization. Proc Natl Acad Sci US A. 2012;109(37):14767-14772.

44. Gekko K, Li X, Makino S. Effects of polyols and sugars on the sol-gel transition of gelatin. Biosci Biotech Biochem. 1992;56(8):1279-1284.

45. Vandermeulen G, Rouxhet L, Arien A, Brewster ME, Preat V. Encapsulation of amphotericin B in poly(ethylene glycol)-blockpoly( $\varepsilon$-caprolactone-co-trimethylenecarbonate) polymeric micelles. Int J Pharm. 2006;309(1-2):234-240.

46. Bang JY, Song CE, Kim C, et al. Cytotoxicity of amphotericin B-incorporated polymeric micelles composed of poly(DL-lactide-coglycolide)/dextran graft copolymer. Arch Pharm Res. 2008;31(11): 1463-1469.

47. Egito LC, de Medeiros SR, Medeiros MG, Price JC, Egito ES. Evaluation of the relationship of the molecular aggregation state of amphotericin B in medium to its genotoxic potential. J Pharm Sci. 2004;93(6):1557-1565.

48. Balakrishnan AR, Easwaran KR. CD and NMR studies on the aggregation of amphotericin-B in solution. Biochim Biophys Acta. 1993;1148(2):269-277. 
49. Barwicz J, Tancrede P. The effect of aggregation state of amphotericin-B on its interactions with cholesterol- or ergosterol-containing phosphatidylcholine monolayers. Chem Phys Lipids. 1997;85(2): 145-155.

50. Huang W, Zhang Z, Han X, et al. Ion channel behavior of amphotericin B in sterol-free and cholesterol- or ergosterol-containing supported phosphatidylcholine bilayer model membranes investigated by electrochemistry and spectroscopy. Biophys J. 2002;83(6): 3245-3255.

51. Puttipipatkhachorn S, Nunthanid J, Yamamoto K, Peck GE. Drug physical state and drug-polymer interaction on drug release from chitosan matrix films. J Control Release. 2001;75(1-2) 143-153.

52. Kai D, Jin G, Prabhakaran MP, Ramakrishna S. Electrospun synthetic and natural nanofibers for regenerative medicine and stem cells. Biotechnol J. 2013;8(1):59-72.
53. Loh XJ, Gong J, Sakuragi M, et al. Surface coating with a thermoresponsive copolymer for the culture and non-enzymatic recovery of mouse embryonic stem cells. Macromol Biosci. 2009;9(11):1069-1079.

54. Loh XJ, Peh P, Liao S, Sng C, Li J. Controlled drug release from biodegradable thermoresponsive physical hydrogel nanofibers. J Control Release. 2010;143(2):175-182.

55. Appel EA, del Barrio J, Loh XJ, Scherman OA. Supramolecular polymeric hydrogels. Chem Soc Rev. 2012;41(18):6195-6214.

56. Ye EY, Loh XJ. Polymeric hydrogels and nanoparticles: a merging and emerging field. Aust J Chem. 2013;66(9):997-1007.

57. Appel EA, Loh XJ, Jones ST, Dreiss CA, Scherman OA. Sustained release of proteins from high water content supramolecular polymer hydrogels. Biomaterials. 2012;33(18):4646-4652.

58. Appel EA, Loh XJ, Jones ST, Biedermann F, Dreiss CA, Scherman OA. Ultrahigh-water-content supramolecular hydrogels exhibiting multistimuli responsiveness. J Am Chem Soc. 2012;134(28):11767-11773. 


\section{Supplementary material}

Activity of antifungal-loaded fiber mats and circular dichroism and rheological studies of gelatin-antifungal interactions.
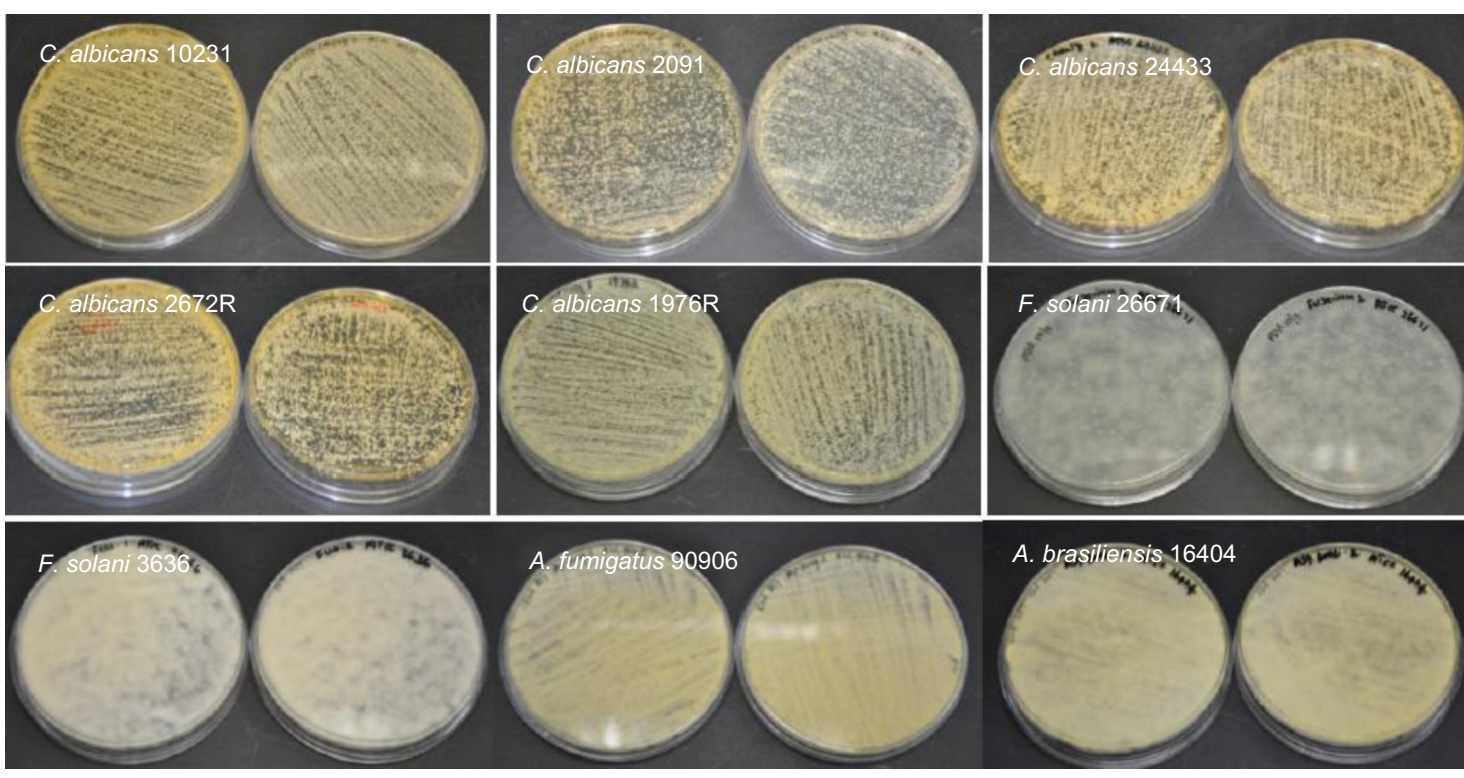

Figure SI Growth of yeast/fungal pathogens on Sabouraud dextrose agar plate.

Abbreviations: C. albicans, Candida albicans; F. solani, Fusarium solani; A. brasiliensis, Aspergillus brasiliensis; A. fumigatus, Aspergillus fumigatus.
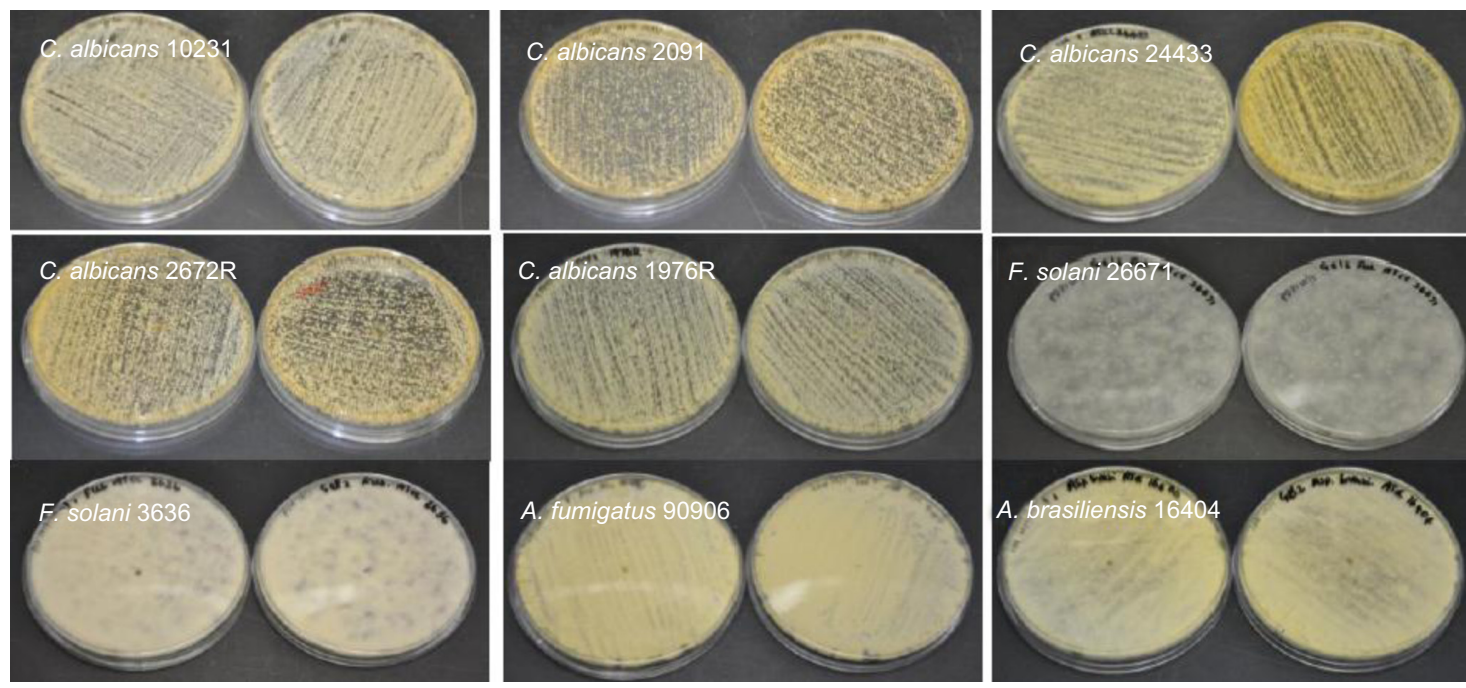

Figure S2 Growth of yeast/fungal pathogens on gelatin fiber mats (without any antifungals).

Abbreviations: C. albicans, Candida albicans; F. solani, Fusarium solani; A. brasiliensis, Aspergillus brasiliensis; A. fumigatus, Aspergillus fumigatus. 

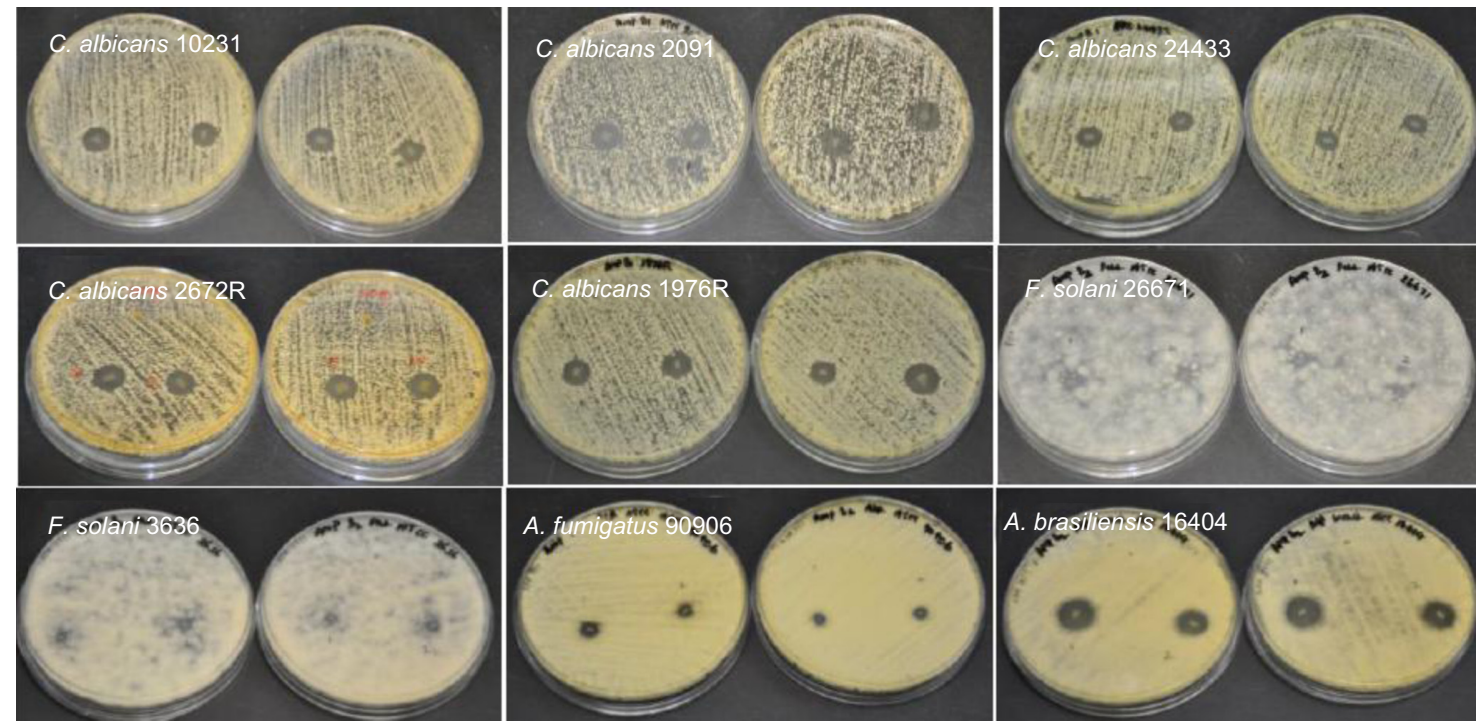

Figure S3 Antifungal properties of amphotericin B-loaded electrospun fiber mats.

Abbreviations: C. albicans, Candida albicans; F. solani, Fusarium solani; A. brasiliensis, Aspergillus brasiliensis; A. fumigatus, Aspergillus fumigatus.
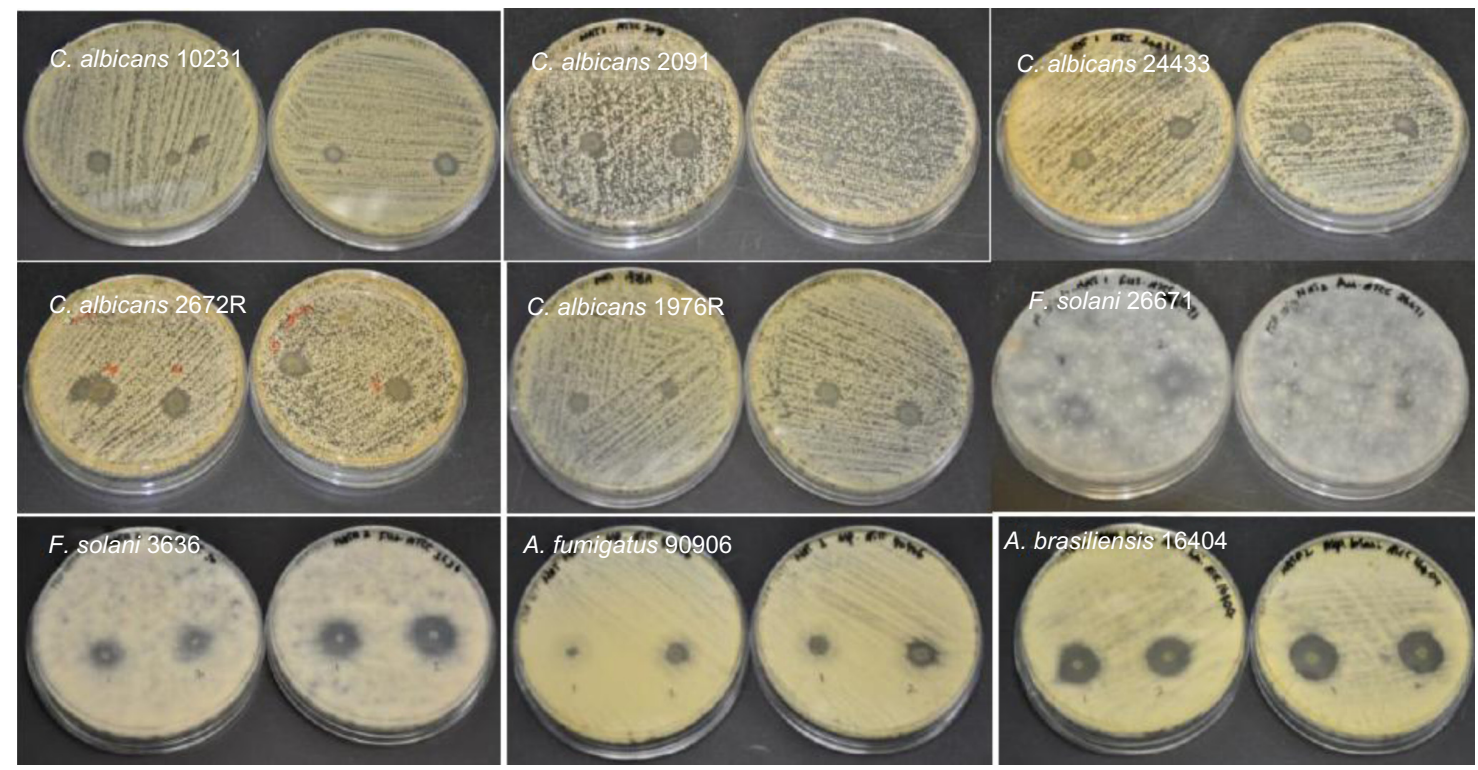

Figure S4 Antifungal properties of natamycin-loaded electrospun fiber mats.

Abbreviations: C. albicans, Candida albicans; F. solani, Fusarium solani; A. brasiliensis, Aspergillus brasiliensis; A. fumigatus, Aspergillus fumigatus. 

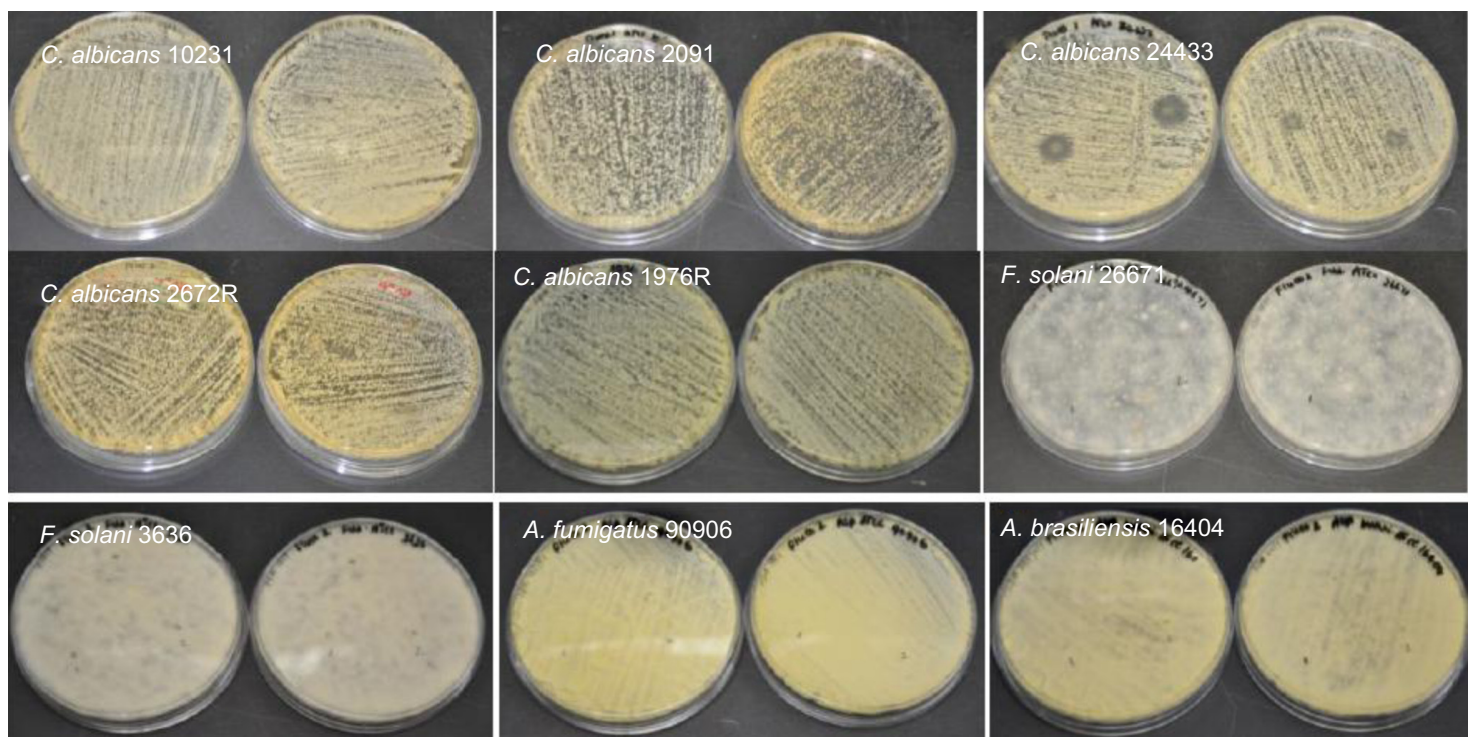

Figure S5 Antifungal properties of fluconazole-loaded electrospun fiber mats.

Abbreviations: C. albicans, Candida albicans; F. solani, Fusarium solani; A. brasiliensis, Aspergillus brasiliensis; A. fumigatus, Aspergillus fumigatus.
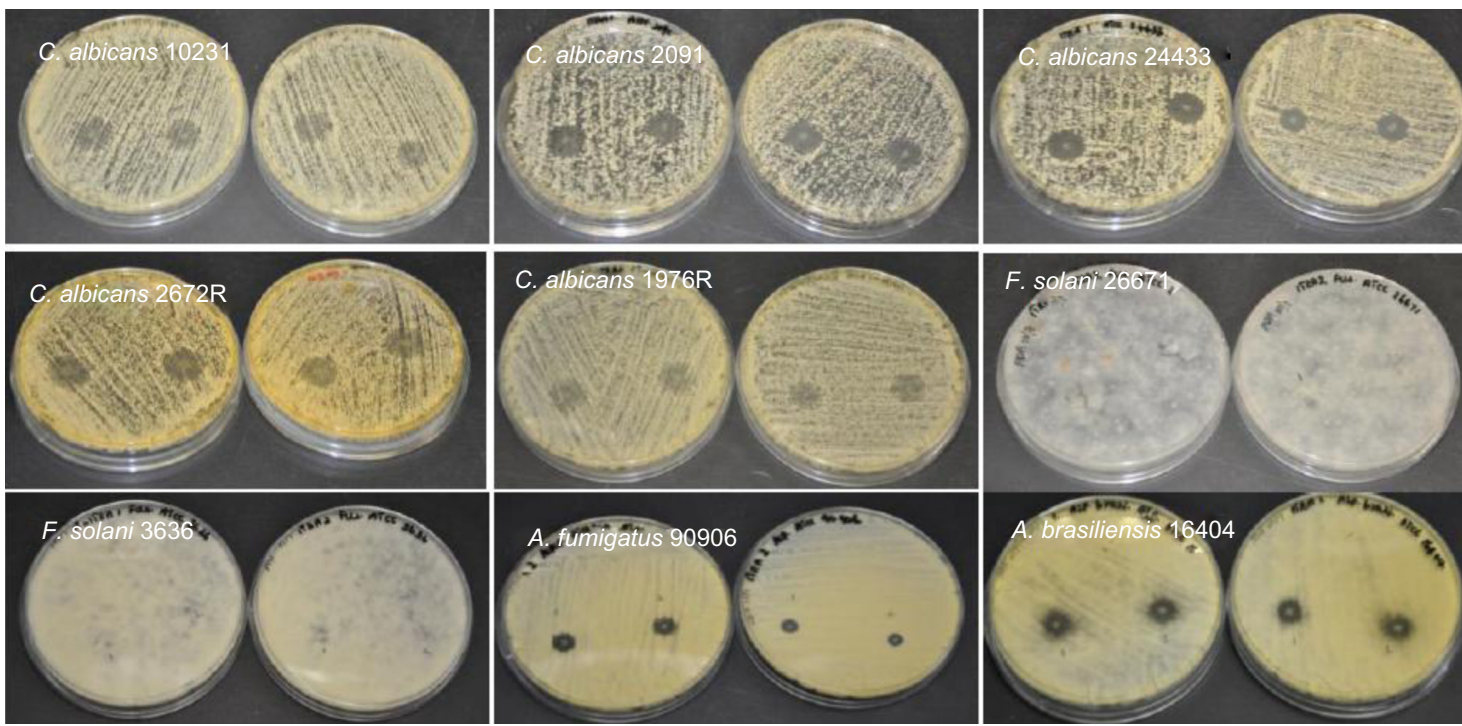

Figure S6 Antifungal properties of itraconazole-loaded electrospun fiber mats. In some strains of $C$. albicans a weak inhibition was observed. Abbreviations: C. albicans, Candida albicans; F. solani, Fusarium solani; A. brasiliensis, Aspergillus brasiliensis; A. fumigatus, Aspergillus fumigatus. 

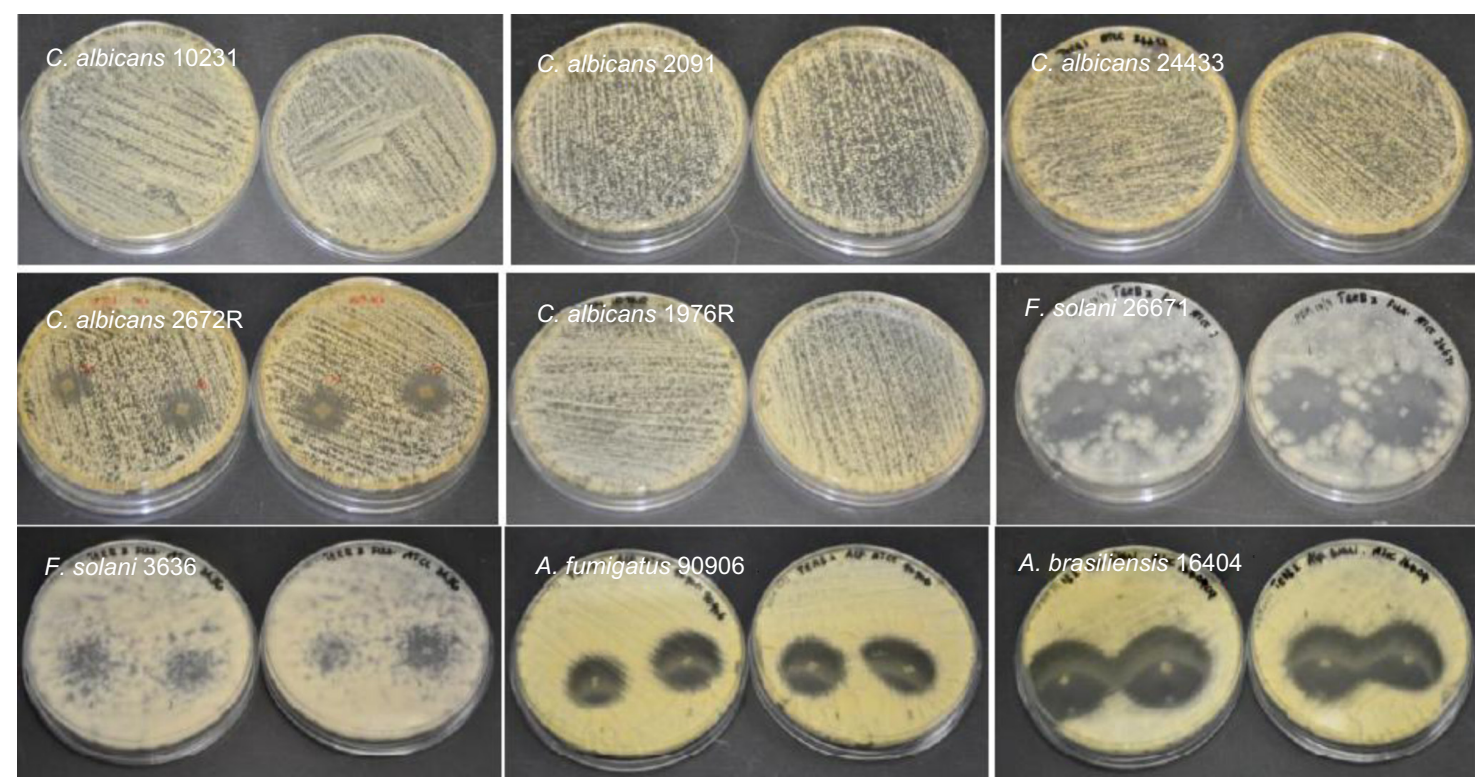

Figure $\mathbf{S 7}$ Antifungal properties of terbinafine-loaded electrospun fiber mats.

Abbreviations: C. albicans, Candida albicans; F. solani, Fusarium solani; A. brasiliensis, Aspergillus brasiliensis; A. fumigatus, Aspergillus fumigatus.
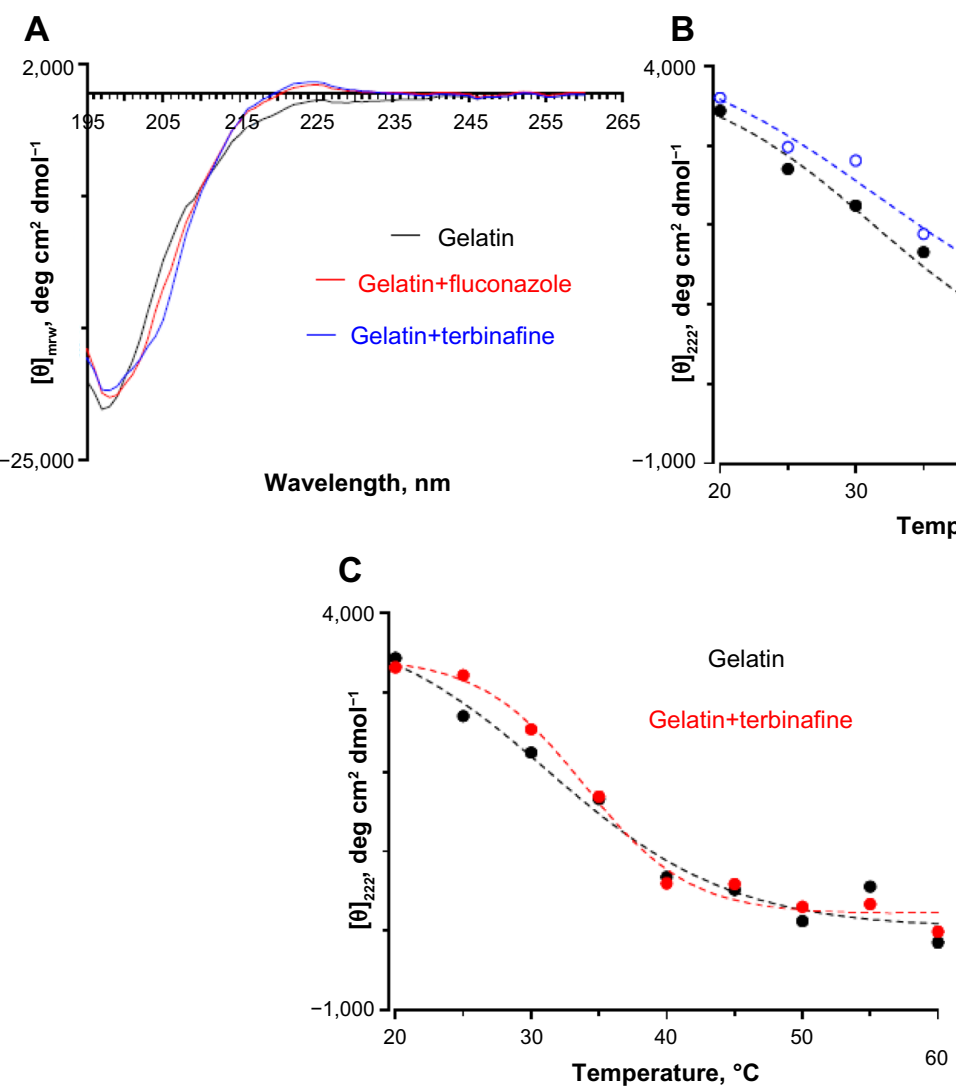

Figure S8 (A) Far ultraviolet circular dichroism spectropolarimetry of gelatin and gelatin incubated with fluconazole and terbinafine at $30^{\circ} \mathrm{C}$. Thermal denaturation of gelatin monitored by changes in $(\theta)_{225}$ in the presence of $(\mathbf{B})$ fluconazole and $(\mathbf{C})$ terbinafine. The concentration of antifungals was 0.25 wt\% with respect to gelatin. 


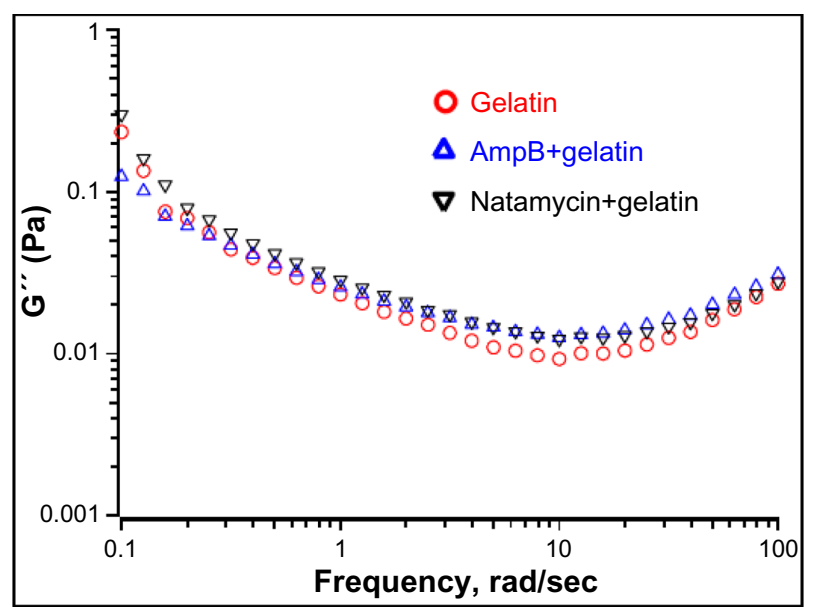

Figure S9 Frequency sweep showing the effect of polyenes on loss modulus ( $\left.\mathrm{G}^{\prime \prime}\right)$. Abbreviation: AmpB, amphotericin B.

\section{Publish your work in this journal}

The International Journal of Nanomedicine is an international, peerreviewed journal focusing on the application of nanotechnology in diagnostics, therapeutics, and drug delivery systems throughout the biomedical field. This journal is indexed on PubMed Central, MedLine, CAS, SciSearch ${ }^{\circledR}$, Current Contents ${ }^{\circledR} /$ Clinical Medicine,
Journal Citation Reports/Science Edition, EMBase, Scopus and the Elsevier Bibliographic databases. The manuscript management system is completely online and includes a very quick and fair peer-review system, which is all easy to use. Visit http://www.dovepress.com/ testimonials.php to read real quotes from published authors.

\footnotetext{
Submit your manuscript here: http://www.dovepress.com/international-journal-of-nanomedicine-journal
} 